\title{
Numerical Simulations of a Flux Rope Ejection
}

\author{
P. Pagano ${ }^{1, *}$, D. H. Mackay ${ }^{1}$ \& S. Poedts ${ }^{2}$ \\ ${ }^{1}$ School of Mathematics and Statistics, University of St Andrews, St Andrews, UK. \\ ${ }^{2}$ Department of Mathematics, Centre for Mathematical Plasma Astrophysics, \\ KU Leuven, Leuven, Belgium. \\ *e-mail:pp25@st-andrews.ac.uk
}

Received 13 August 2014; accepted 10 January 2015

\begin{abstract}
Coronal mass ejections (CMEs) are the most violent phenomena observed on the Sun. One of the most successful models to explain CMEs is the flux rope ejection model, where a magnetic flux rope is expelled from the solar corona after a long phase along which the flux rope stays in equilibrium while magnetic energy is being accumulated. However, still many questions are outstanding on the detailed mechanism of the ejection and observations continuously provide new data to interpret and put in the context. Currently, extreme ultraviolet (EUV) images from the Atmospheric Imaging Assembly (AIA) on board the Solar Dynamic Observatory (SDO) are providing new insights into the early phase of CME evolution. In particular, observations show the ejection of magnetic flux ropes from the solar corona and how they evolve into CMEs. However, these observations are difficult to interpret in terms of basic physical mechanisms and quantities, thus, we need to compare equivalent quantities to test and improve our models.
\end{abstract}

In our work, we intend to bridge the gap between models and observations with our model of flux rope ejection where we consistently describe the full life span of a flux rope from its formation to ejection. This is done by coupling the global non-linear force-free model (GNLFFF) built to describe the slow low- $\beta$ formation phase, with a full MHD simulation run with the software MPI-AMRVAC, suitable to describe the fast MHD evolution of the flux rope ejection that happens in a heterogeneous $\beta$ regime. We also explore the parameter space to identify the conditions upon which the ejection is favoured (gravity stratification and magnetic field intensity) and we produce synthesised AIA observations (171 $\AA$ and $211 \AA$ ). To carry this out, we run 3D MHD simulation in spherical coordinates where we include the role of thermal conduction and radiative losses, both of which are important for determining the temperature distribution of the solar corona during a CME.

Our model of flux rope ejection is successful in realistically describing the entire life span of a flux rope and we also set some conditions for the backgroud solar corona to favour the escape of the flux rope, so that it turns into a CME. Furthermore, our MHD simulation reproduces many of the features found in the AIA observations. 


\section{P. Pagano et al.}

Key words. Coronal mass ejections—-magnetohydrodynamics—simulations-corona.

\section{Introduction}

Coronal Mass Ejections (CMEs) are violent eruptions of plasma and magnetic flux from the solar corona. They have been observed since 1971 (Tousey 1973) with increasingly more accurate and precise instruments and described with more and more sophisticated theoretical models. However, in spite of the observational and theoretical efforts, many aspects of CMEs remain an enigma. One of the most recent satellites to study CMEs is the Solar Dynamic Observatory (SDO) and, in particular, the Atmospheric Imaging Assembly instrument (AIA; Lemen et al. (2012)) continuously observes the full solar disk providing observations in extreme ultraviolet (EUV) channels. Images from AIA enable us to observe the structures from which CMEs originate, the regions where they are produced, and the early phase of ejections, with high resolution and high cadence. At the same time, more complex and more accurate theoretical models have been developed and, while the great majority of them are based on Magnetohydrodynamics (MHD) theory, also small-scale processes and non-ideal MHD effects need to be taken into account in order to tackle the scientific challenge. Additionally, because of the complexity of these models, theoreticians more and more resort to numerical simulations that have become the most common investigation tool for models of the generation and propagation of CMEs. To bridge the gap between observations and models, a key step is to identify which features are understood by our models and which are not. To do that, we need to compare equivalent quantities from theoretical models and observations.

Although the connection between flux ropes and CMEs is not unequivocally determined, flux rope ejections are considered the main CME precursor. Magnetic flux ropes (or just flux ropes) are idealized structures useful to describe many features observed in the solar corona, e.g. sigmoids, filaments, prominences, which are basically constituted by magnetic field lines connecting two footpoints on which the coronal plasma lies. Flux ropes form in the solar corona due to the action of photospheric motions, flux cancellation and flux emergence. Some statistical studies have associated the ejection of a flux rope with at least $40 \%$ of CMEs (Vourlidas et al. 2013). This should be considered a lower boundary as, for the remaining $60 \%$ of CMEs, we do not have sufficient information. Therefore, it is crucial to study and understand the process of flux rope ejections to properly describe the early stages of a CME. The complexity of the equations and the different physical regime involved made numerical simulations the privileged tool for theoreticians to develop models of flux rope ejections. Once formed, a flux rope normally lies in the solar corona in a state near equilibrium. This phase can last from some hours, up to months and some small scale processes such as wave propagation or coronal rain can be observed. At the end, the flux rope may be ejected and when this happens, the ejection phase lasts a few hours and ends with the flux rope leaving the solar corona. During this phase, the system is always far from equilibrium. How this transition between equilibrium and ejection occurs is the key question to be answered if we want to understand flux rope ejections. Chen (2011) made an extensive review on the physics of CME, where specific models of flux rope ejection have also been addressed. We refer to this review for details. In some models, the ejection is triggered by processes enabled by 
bulk motions of plasma either on the solar surface or from below it, whereas in other models the ejection is triggered from a standing flux rope. A number of numerical models successfully describe the main features of flux rope ejections starting from an undisturbed flux rope lying in the solar corona. These models usually require that the equilibrium is unstable or that a spontaneous diffusive process (flux cancellation or magnetic reconnection) takes place, altering the equilibrium of the structure. Sakurai (1976) uses an analytical model to suggest that the ejection of a flux rope can be due to the development of a kink instability and, more recently, numerical simulations described the evolution of such a process in realistic terms for the solar corona. Török and Kliem (2005) reproduced a quenched ejection of flux rope observed with Transition Region and Coronal Explorer (TRACE) and concluded that although the kink instability is able to onset the ejection, if the overlying magnetic field does not decay quickly enough, the ejection fails. Inoue and Kusano (2006) posed some constraints on the length of the flux rope, but concluded that the kink instability can lead to the full ejection of a flux rope. Kliem et al. (2010) showed the dynamics of an ejection initiated by the kink instability and clarified how this specific instability needs favourable circumstances such as fast decay of the overlying magnetic field (torus instability) or high twist of the flux rope that favours leg-leg reconnection. Following this study, a parameter space investigation by Kliem et al. (2012) showed that only with a moderate initial twist of the flux rope the ejection has a rotation that matches observations. These investigations have analysed many realistic cases and, although it is not possible to say a final word, the kink instability is a viable mechanism that can concur to initiate an ejection, but it is not a necessary nor sufficient condition for this to happen.

Magnetic flux ropes are usually embedded in arcade systems, so the torus instability has been indicated as a possible cause for the ejection. In simple terms, the torus instability occurs when the intensity of the overlying magnetic field decays too steeply with height and the hoop force is not able to constrain the flux rope down. It was introduced in the domain of coronal physics by Chen (1989) and Kliem and Török (2006) and, since then, a number of numerical investigations have been carried out. In many studies the torus instability is effectively responsible for the ejection of the flux rope, but the flux rope needs to be initially displaced to a region where the decay index of the arcade field is steep enough or a slow reconfiguration of the magnetic field needs to happen to make the overlying arcade torus unstable. We will discuss these numerical models later on. In some studies, such as Török and Kliem (2005) or Kliem et al. (2010), the torus instability is an inherent property of the magnetic configuration under investigation and determines the conditions for the ejection, although it is not the triggering mechanism. Kliem et al. (2013) tuned a simulation of the ejection that occurred on 2010 April 8, starting from a magnetic configuration torus unstable generated by real magnetograms and the simulation reproduces many characteristics of the observed eruption such as the direction of propagation, the accelaration, and the temporal sequence between acceleration and reconnection. Subsequently, Kliem et al. (2014) completed a numerical and analytical study, where they bridged the torus instability scenario with the catastrophe model (Priest \& Forbes 1990; Forbes \& Isenberg 1991). This last work simplifies the classification of flux rope ejection models and makes it possible to ascribe to this class the many models developed from the catastrophe models. Nishida et al. (2013) developed a 3D MHD simulation of an infinite flux rope lying on the solar corona. 


\section{P. Pagano et al.}

The flux rope is ejected because of the loss of equilibrium due to kink and torus instability and they found that $3 \mathrm{D}$ effects play an important role in determining the ejection properties.

In another class of numerical models the flux rope is ejected because the magnetic field reconnects and this alters the force balance. Examples of this scenario are the flux cancellation models, where the magnetic field reconnects under the flux rope at the polar inversion line and this leads to an increased Lorentz force that pushes the flux rope outwards. Although the flux cancellation underneath the flux rope is usually the consequence of a structured photospheric motion, there are some examples of numerical studies where the photospheric motion is not artificially prescribed and organized to enhance flux cancellation. Yeates and Mackay (2009) carried out a complex modelling of the evolution of the global magnetic field in the solar corona based on the magnetofrictional relaxation of the non-linear force free field reconstruction from magnetograms constantly stressed by differential rotation and meridional flow. In this approach, although some photospheric flows are present, they are not built to specifically generate flux rope ejections that eventually occur over the simulation. Inoue et al. (2014) run numerical simulations starting from a non-linear force free extrapolation of magnetic field from the magnetograms associated to the solar flare which occurred on 2011 February 15. In this work, the ejection is enabled by the use of an anomalous magnetic resistivity acting where the electric current is greater than a set threshold. The thether-cutting process following the flux cancellation results are crucial to enhance the ejection and the MHD simulation roughly reproduces the EUV image from AIA of that flare. Kusano et al. (2012) conducted numerical simulations, where two sheared arcades are in contact and the ejection is onset when the relative position is favourable to flux cancellation.

In the break-out model and its following version, the confining force that holds down the flux rope is removed via magnetic reconnection. In its original version of Antiochos et al. (1999), the presence of a null-point above the flux rope was crucial to initiate the confinement removal and the subsequent ejection. The breakout model can be considered a specific case of a larger class of models, where the ejection is triggered by the removal of magnetic confinement from a region overlying the flux rope. Shiota et al. (2010) performed a global numerical simulation of the solar corona where a flux rope interacts with the ambient magnetic field. Although they do not specifically address the trigger mechanism, they conclude that the interaction between the flux rope and ambient magnetic field plays a crucial role in determining the fate of the ejection. They, interestingly, argue that the reconnection ahead of the ejection may indeed favour the ejection, but also dissipate the flux rope. More recently, Jiang et al. (2013) performed an advanced modelling of the active region AR 11283 where a sigmoid was observed to erupt. In this study, the ejection occurs because a null point is present above the sigmoid and the magnetic confinement is removed as the ejection goes on. A comparison of the numerical simulation with AIA observation shows a good matching, making the interpretation of results significant. Török et al. (2011) presents a complex configuration where four flux ropes are initially lying in equilibrium in the solar corona and where the ejection of the first one allows the reconnection and the subsequent removal of magnetic confinement for two others.

Another large class of models are those that associate the flux rope ejection to photospheric motions. The nature of these numerical models is such that the 
photospheric motions are used in combination with other physical processes such as magnetic reconnection or flux cancellation. To this end, photospheric motions are not the final trigger, but they are the initiator of the chain of events that leads to the flux rope ejection. The solar corona is patched by bundles of coronal loops that connect different regions. With this pattern in mind, it is natural to imagine that when the footpoints of these bundles are drifted, the system accumulates stress that needs to be released afterwards. The stretching or compression of magnetic field lines can certainly lead to the formation of new structures as flux ropes and alter the existing force balance. Following the intuition of van Ballegooijen and Martens (1989), magnetic field lines stretched by shearing motions can reconnect to build a flux rope and the resulting structure is pushed outwards. Earlier, Mikic and Linker (1994) numerically simulated this process and a number of works followed thereafter. Amari et al. (2003a, b) also addressed the role of magnetic helicity and explained how the flux rope can form during the ejection. Jacobs et al. (2006), in a 2.5D numerical simulation, accounted for both shearing motions and solar wind outlining - a relation between the strength of the shearing and the speed of the resulting CME. Kusano et al. (2004) described how shear reversal in the solar corona can lead to largescale eruption when it sets on resistive-tearing mode instabilities. Aulanier et al. (2010) made a detailed analysis of the dynamics of a flux rope ejection, where the initial stress is accumulated through shearing motions. In this work, the shearing of the footpoints is followed by the flux cancellation underneath the flux rope, the displacement of the flux rope in a region torus unstable, and finally the magnetic reconnection feeds back the ejection. Although the whole process starts with the shearing motions, it is an example on how several mechanisms can concur to the flux rope ejection. Building on this work, Savcheva et al. (2012) shows a comparison between the NLFFF extrapolations from the magnetograms associated to the CME observed on 2007 February 12 and a MHD numerical simulation, where the ejection is reproduced from an initially bipolar configuration that undergoes the ejection mechanism of Aulanier et al. (2010). The two approaches, although different, consistently describe the main characteristics of the observations.

Alternatively, the foot points motion can build up stress in different ways than moving away from each other: in the simulation of Amari et al. (2011) the flux cancellation is enhanced by converging flows at the Polar Inversion Line (PIL) under the flux rope. Roussev et al. (2004) simulated the evolution of the coronal mass ejection that occurred that on 1998 May 2 in NOAA Active Region 8210, by means of converging motions that simulate the effect of flux cancellation. Zuccarello et al. (2012) triggered a flux rope ejection with the rotation in opposite directions of the footpoints of a bipolar region reconstructed from magnetograms of AR NOAA 9415. Török et al. (2013) tuned a numerical simulation to reproduce an observed magnetic configuration in NOAA Active Region 10898 on 2006 July 6, where a flux rope is present. The rotation of the sunspot about the flux rope onsets the CME.

Another way how the flux rope ejections are initiated by the interaction between the solar corona and subphotospheric layers, is when magnetic flux emerges from under the photosphere. When this happens, the newly emerged magnetic flux can potentially disrupt the coronal equilibrium and solar eruptions occur. Several numerical experiments have been carried out to explain this specific coupling between the convective layers and the solar corona. Fan (2005) initiated a series of numerical studies in spherical geometry, where a kinked flux rope emerges in the solar corona. 


\section{P. Pagano et al.}

When the flux rope has emerged, the kink instability develops, producing a CME. In a subsequent work, they found that the same process can lead to a torus instability still resulting in a CME (Fan \& Gibson 2007). Fan (2010) developed a parameter space investigation where different amounts of flux are emerged and found that the onset of an ejection happens when the flux rope reaches a critical height during the emerging phase. Chatterjee and Fan (2013) explain how a cannibalistic CME can result from the overposition of several failed ejections during flux emergence. Archontis and Török (2008) found that when an emerged flux rope is allowed to reconnect with the coronal field, a process similar to the breakout starts and the flux rope erupts. MacTaggart and Hood (2009) described that while a toroidal flux rope emerges, multiple eruptions can take place, and, subsequently, a numerical simulation in Archontis et al. (2014) showed a similar result with a cylindric flux tube. The numerical simulation developed in Roussev et al. (2012) describes how the global reorganization of the solar corona that follows from the emergence of flux generates CME and how reconnection can enhance fast CMEs. Leake et al. (2014) describe that a CME can develop from a flux emergence event only if the flux rope is allowed to reconnect with the external field, but the major acceleration of the flux rope occurs when also internal reconnection is onset, which plays the role of the flux cancellation. All these models give present plausible explanation of the mechanism that triggers the ejection of a flux rope. Although so far none of these has been clearly ruled out, observations will play a crucial role in identifying the processes occurring in the Sun.

In this work, we outline the main characteristics of the approach developed starting from the work of Pagano et al. (2013a), where the phases of flux rope formation and flux rope ejection are coupled, but separately modelled. The flux rope formation coincides with the phase when energy is accumulated as the magnetic field slowly departs from its lower energy state. This happens very slowly (days to weeks or months) and the magnetic field evolves through a series of equilibrium states (Forbes 1991; Mackay \& van Ballegooijen 2006a, b; Yeates \& Mackay 2009; Yeates et al. 2010). In contrast, when the flux rope is ejected, equilibrium does not hold, plasma is compressed and heated to an extent where regions at high $\beta$ develop, and the eruption ends in a few hours. The variety of time scales involved poses a considerable challenge to theoretical models, so our strategy is to use the global non-linear force-free field model of Mackay and van Ballegooijen (2006a) (hereafter called GNLFFF) which neglects the role of the plasma for the formation of the flux rope in the zero- $\beta$, quasi-static approximation and, once the flux rope forms and is about to erupt, we switch to a full MHD approach, where the stressed magnetic field configuration from the GNLFFF model is let to evolve in MHD simulations with the MPI-AMRVAC code (Porth et al. 2014). In this way, we can accurately describe the full life span of a flux rope from its slow generation to its rapid ejection.

Once we have proved to have an eruptive configuration, we can assess the role of the background corona in quenching or favouring the flux rope ejection (Pagano et al. $2013 b$ ). In particular, we address the role of gravitational stratification and $\beta$ regime. This is done by running a set of MHD simulations, where the temperature of the background corona and the intensity of the magnetic field vary, to understand when a flux rope ejection can turn into a CME. This is specifically important for our space weather forecast ambition, where we need to know precisely the velocity, trajectory 
and energy of each ejection. From another point of view, an increased understanding of the coupling between the flux rope ejection and the background corona can result in using ejecting flux ropes as probes that can reveal thermodynamics parameters of the plasma encountered on their motion.

Finally, in order to bridge the gap between model and observations, we synthesize AIA observations from our MHD simulations, as to compare modelled features with actually observed flux rope ejections. A similar attempt has been carried out in Pagano et al. (2014). AIA observations have greatly enhanced our understanding of flux rope ejections and any model must be confronted with actual observations via homogeneous quantities, such as synthesised observations. Here, we present the synthesis of $171 \AA$ channel, which is sensible to the low temperature component of the solar corona and $211 \AA$ channel, which is useful to highlight the feedback of the flux rope ejection of the solar surface.

The paper is structured as follows. In section 2, we describe the general setup of the simulations. In section 3, we describe the physical mechanism that leads to the ejection of the flux rope. In section 4, we determine the parameter space where the ejections are likely to occur. In section 5, we show the synthesis of AIA observations from our simulation. Finally, in section 6, we draw some conclusions from this study.

\section{Method}

In order to model the full life span of a flux rope from the slow quasi-static builtup to the rapid ejection, we adopt two different numerical models for the formation and ejection phases. The output of one model is used as the initial condition for the other. Whereas this is the general strategy, the coupling of the two models require a number of technical arrangements that needs a detailed explanation, to understand the potential and limitations of our approach. Two main aspects need to be considered: (i) when importing a magnetic configuration from one model to another, some spatial interpolation is needed, as two models do not necessarily work in the same space discretization framework, (ii) in order to switch from a zero- $\beta$ approximation model to a full MHD model, we need to adopt a thermodynamic description for the coronal plasma that completes the set of variables of the GNLFFF simulation for the MPI-AMRVAC MHD simulation.

We describe in section 2.1, how the two models are coupled, and in section 2.2, how we include the coronal plasma in the setup. In section 2.3, we describe how we advance the simulation, i.e. the MHD equations.

\subsection{Coupling of the two models}

The initial condition of the model of Mackay and van Ballegooijen (2006a) consists of two nearby bipoles, where one bipole is slightly more tilted with respect to the equator than the other. The two bipoles are then advected under the effect of solar differential rotation, meridional flow and surface diffusion. Under the effect of photospheric motion, the bipoles get sheared and a flux rope forms above the PIL of the more tilted bipole after 19 days of evolution (Figure 1). We identify this as the time 


\section{P. Pagano et al.}

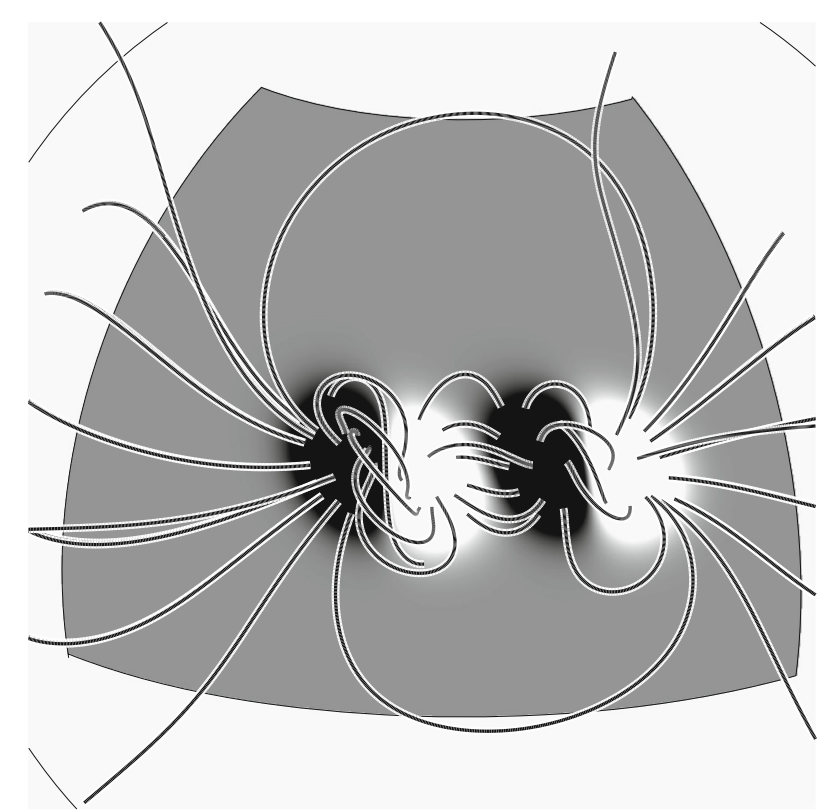

Figure 1. Configuration of the magnetic field after 19 days of evolution using the model of Mackay and van Ballegooijen (2006a). The figure shows a sample of magnetic field lines that illustrate the structure of the magnetic bipoles, the overlying arcade, and the newly formed magnetic flux rope along the PIL of the left-hand side bipole. The surface boundary is coloured according to the polarity of the magnetic field: negative polarity is black and positive is white.

when a flux rope has clearly formed in the simulation and the corresponding magnetic configuration is used as the initial magnetic field condition for numerical MHD simulations using MPI-ARMVAC.

The coupling of the two models is a non-trivial process. To begin with, only the magnetic field, $\vec{B}$, is a common variable to both models. The three components of the magnetic field are, therefore, directly transported from the GNLFFF code to the MHD code. The transport is designed to preserve the gradients of $\vec{B}$, thus, the forces in the system. In the example shown here, we have two grids with a different number of points and different spacing: the GNLFFF grid is composed of $106 \times 172 \times 181$ cells, where the cell size is non-uniform (see Mackay \& van Ballegooijen (2006a)) and the MPI-AMRVAC grid is composed of $132 \times 128 \times 128$ cells uniformly spaced. As the black and red cells in Figure 2 are of a different size they do not match, therefore, the value of $\vec{B}^{V A C}$ needs to be interpolated at each point. First, we identify the cell of the GNLFFF grid into which each point of the MHD grid falls and then we use the values of the adjacent GNLFFF cells for the computation of the interpolated magnetic field:

$$
\begin{aligned}
\vec{B}_{[r, \theta, \phi]}^{V A C}\left(k_{r}, j_{\theta}, i_{\phi}\right)= & \vec{B}_{[r, \theta, \phi]}^{\mathrm{GNLFFF}}\left(r_{0}, \theta_{0}, \phi_{0}\right) \\
& +\nabla \vec{B}_{[r, \theta, \phi]}^{\mathrm{GNLFFF}} \cdot \overrightarrow{\Delta s}+\frac{1}{2} \overrightarrow{\Delta s}^{T} \cdot H^{\mathrm{GNLFFF}}\left(r_{0}, \theta_{0}, \phi_{0}\right) \cdot \overrightarrow{\Delta s}
\end{aligned}
$$




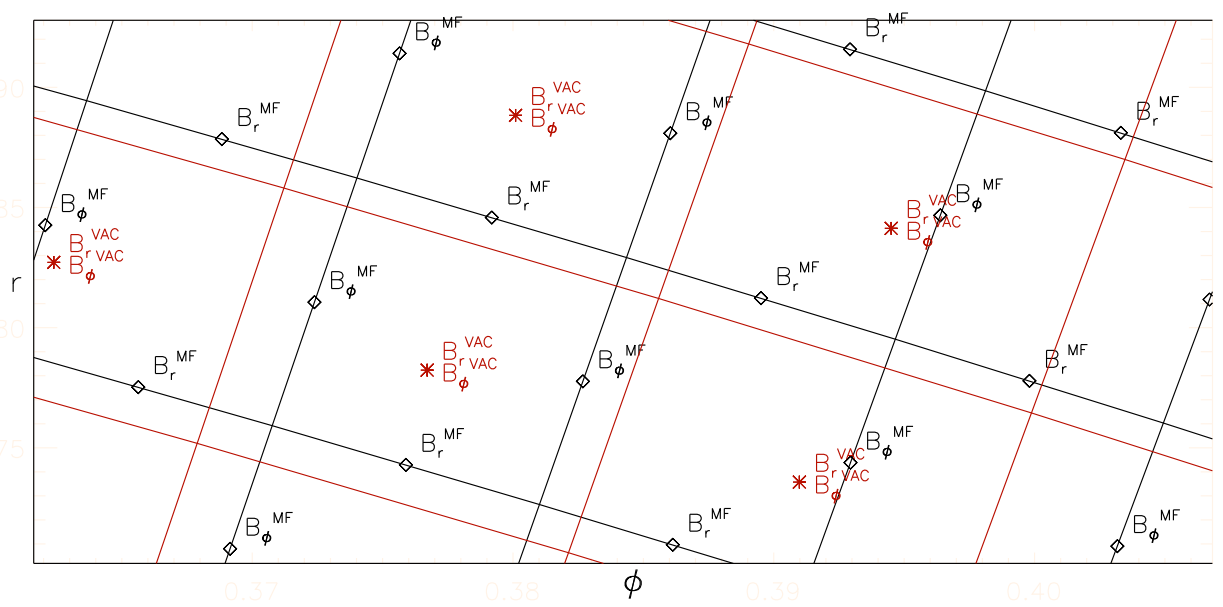

Figure 2. An illustration of a portion of the $r-\phi$ plane on to a flat surface where we sketch the two grids. Black lines represent the GNLFFF code: the magnetic field components are defined on different ribs of the cells. Red lines represent the cells of AMRVAC: all the magnetic field components are defined in the centre of the cell.

where $k_{r}, j_{\theta}, i_{\phi}$ are the indices identifying the position in the MHD grid and $\vec{B}_{[r, \theta, \phi]}^{\mathrm{GNLFF}}\left(r_{0}, \theta_{0}, \phi_{0}\right)$ are the values of the components of $\vec{B}^{\mathrm{GNLFFF}}$ closest to the coordinates of the point $\left(k_{r}, j_{\theta}, i_{\phi}\right)$ and $\overrightarrow{\Delta s}=(\mathrm{d} r, r \mathrm{~d} \theta, r \sin \theta \mathrm{d} \phi)$. The adjacent cells are used to compute the gradients of the components of the magnetic field, $\nabla \vec{B}_{[r, \theta, \phi]}^{\mathrm{GNLFF}}$ and the Hessian matrix $\left|H^{\mathrm{GNLFFF}}\left(r_{0}, \theta_{0}, \phi_{0}\right)\right|$ through finite differences. The third order interpolation (second order on the derivatives) is performed as it guarantees a correct transporting of the spatial derivatives of $\vec{B}$ between the codes and thus the Lorentz Force. The components of the interpolated magnetic field (MHD simulation) depart by at most $1 \%$ from their original value in the GNLFFF model. This occurs where the spatial gradients of the magnetic field components are the steepest.

The computational domain in the GNLFFF model spans from the solar surface to a height of $2.5 R_{\odot}$. The colatitude, $\theta$, spans from $\theta=30^{\circ}$ to $\theta=100^{\circ}$ and the longitude, $\phi$, spans over $90^{\circ}$. In some simulations, we extend the computational domain to $4 R_{\odot}$ and in these cases we assume a pure radial magnetic field ( $B_{\theta}=$ $B_{\phi}=0$ ) for $r>2.5 R_{\odot}$, where the magnetic flux is conserved

$$
B_{r}\left(r>2.5 R_{\odot}, \theta, \phi\right)=B_{r}\left(2.5 R_{\odot}, \theta, \phi\right) \frac{2.5^{2}}{r^{2}} .
$$

In order to ensure that the coupling of the two models does not create spurious forces that could generate artificial motions, we have first tested that a force-free magnetic configuration remains stable also in the MHD simulation. Our tests show that in such circumstances, the magnetic configuration does not show any significant evolution after $\sim 100$ Alfvén times and changes to the magnetic field are of the order of $1 \%$ of the initial value (Pagano et al. 2013a). 


\section{P. Pagano et al.}

\subsection{Atmosphere}

In order to run a MHD simulation, we need to model the plasma distribution of the corona in which the imported magnetic configuration is merged. The variables to be added from the GNLFFF model input are velocity and two variables among plasma density, thermal pressure, and temperature (we choose plasma density and thermal pressure). As the velocity, $\vec{v}$, in the GNLFFF model is a non-physical relaxation velocity, we do not import it into the MHD simulation and we choose to initially set $\vec{v}=0$, as we believe to start from a near-equilibrium state. As far as density and pressure are concerned, we use two different approches. A simple one for the sole purpose of testing whether the magnetic configuration is eruptive, and another, one more complex whose aim is at providing a realistic atmosphere to be used for the synthesis of AIA observations.

2.2.1 Constant pressure atmosphere. In the simple approach, we adopt a background atmosphere where no gravitational stratification and gravity are considered. We assume a density of the plasma distribution, $\rho$, that gives a nearly constant Alfvén speed all over the domain:

$$
\rho(r, \theta, \phi)=\rho_{0} \frac{B(r, \vec{\theta}, \phi)^{2}}{B_{0}^{2}}+\rho_{\mathrm{bg}}
$$

where we include a background uniform distribution, $\rho_{\mathrm{bg}}$, to avoid extremely low density values occurring where the magnetic field is weak and $\rho_{0}$ and $B_{0}$ are the normalization values to tune the final density distribution $\rho(r, \theta, \phi)$. We also assume a uniform thermal pressure, $p$,

$$
p=p_{0},
$$

where $p_{0}$ is a constant value. In this simple setting, no other force than the Lorentz force initially acts on the system, as no gravity and no pressure gradient are present.

2.2.2 Atmosphere with flux rope. We aim to study the effect of the background corona or at reproducing the EUV emission of the coronal plasma during a flux rope ejection, hence, we need a realistic modelling of the coronal atmosphere. In order to do so, we prescribe a non-isothermal solar corona. To produce the temperature distribution, we start from the auxiliary function $\sigma(\vec{B})$, which is the magnetic field dependent temperature (except for some small deviations):

$$
\sigma(\vec{B})=\left[\left(\frac{6}{2+B_{\theta} /|B|}-2\right)\left(\sigma_{\text {out }}-\sigma_{\text {min }}\right)+\sigma_{\min }\right](1-G(\vec{B}))+\sigma_{\text {out }} G(\vec{B}),
$$

where $\sigma_{\text {out }}$ is the value of $\sigma(\vec{B})$ when $B_{\theta}=0$ and $G(\vec{B})=0$. The parameter $\sigma_{\text {min }}$ determines the minimum allowed value for $\sigma(\vec{B})$, where $B_{\theta}=|B|$ and $G(\vec{B})=0$. The function $G(\vec{B})$ is used to bound the dependence of $\sigma$ on $\vec{B}$ to the regions where the magnetic field strength is significant:

$$
G(\vec{B})=\mathrm{e}^{-\frac{|B|^{2}}{2 B_{*}^{2}}}
$$


A typical value for $B_{*}$ is $B_{*}=3 G$ in our simulations. This is an ad hoc analytic formula that is justified in our setting, as flux rope lies in the $\theta$ direction with a positive $B_{\theta}$ and it is the only such structure. The thermal pressure distribution is independently specified using the solution for hydrostatic equilibrium with uniform temperature set equal to $\sigma_{\text {out }}$,

$$
p=\frac{\rho_{L B}}{\mu m_{\mathrm{p}}} k_{\mathrm{b}} 2 \sigma_{\text {out }} \mathrm{e}^{\frac{M_{\odot} G \mu m_{\mathrm{p}}}{2 \sigma_{\sigma_{\text {out }}}}\left(\frac{1}{r}-\frac{1}{R_{\odot}}\right)}+\frac{\rho_{\mathrm{bg}}}{\mu m_{\mathrm{p}}} k_{\mathrm{b}} 2 \sigma_{\text {out }},
$$

where $\rho_{L B}$ is the density at $r=R_{\odot}$ when $|B|=0, \mu=1.31$ is the average particle mass in the solar corona, $m_{\mathrm{p}}$ is the proton mass, $k_{\mathrm{b}}$ is Boltzmann constant and $\rho_{\mathrm{bg}}$ is a background constant density that prevents ultra-low values of the plasma $\beta$ in the outer corona. Finally, the density is simply given by the equation of state applied to eq. (5) and eq. (7) (using $\sigma(\vec{B})$ as temperature) along with the background density term:

$$
\rho=\frac{p}{\sigma(\vec{B})} \frac{\mu m_{\mathrm{p}}}{k_{\mathrm{b}}} .
$$

Note that the temperature, $T$, in our MHD simulation is independently derived from the thermal pressure, $p$, and the density, $\rho$, and it identically coincides with $\sigma(\vec{B})$ when $\rho_{\mathrm{bg}}=0$ : the higher the $\rho_{\mathrm{bg}}$, the lower the temperature in the MHD simulation. Since the temperature distribution is proportional to $\sigma(\vec{B})$, the magnetic flux rope (where $B_{\theta} \approx|B|$ ) is colder than the external corona. In some simulations, we need to have non-zero values for $\rho_{\mathrm{bg}}$ to avoid extremely low $\beta$ values at the outer radial boundary of the simulation. In our simulations such a departure from equilibrium implies a negligible inflow of plasma (in contrast to the real solar corona that is outflowing). When we set $\sigma_{\text {out }}=\sigma_{\min }$ and $\rho_{\mathrm{bg}}=0$ we are in the case of an isothermal stratified atmosphere and the function $\sigma(\vec{B})$ coincides with the plasma temperature $T$. In general, this approach is suitable to realistically describe the region surrounding a flux rope, but becomes less accurate at higher radial distance from the Sun where the solar wind plays an important role in shaping the solar atmosphere.

\subsection{Equation}

Once the initial condition of the simulation is set, we run the MPI-AMRVAC software (Porth et al. 2014; van der Holst et al. 2008), to solve the MHD equations. We can also include external gravity, anisotropic thermal conduction, and optically thin radiative losses as source terms:

$$
\begin{gathered}
\frac{\partial \rho}{\partial t}+\vec{\nabla} \cdot(\rho \vec{v})=0, \\
\frac{\partial \rho \vec{v}}{\partial t}+\vec{\nabla} \cdot(\rho \vec{v} \vec{v})+\nabla p-\frac{(\vec{\nabla} \times \vec{B}) \times \vec{B}}{4 \pi}=+\rho \vec{g}, \\
\frac{\partial \vec{B}}{\partial t}-\vec{\nabla} \times(\vec{v} \times \vec{B})=0,
\end{gathered}
$$




$$
\frac{\partial e}{\partial t}+\vec{\nabla} \cdot[(e+p) \vec{v}]=\rho \vec{g} \cdot \vec{v}-n^{2} \chi(T)-\nabla \cdot \vec{F}_{c},
$$

where $t$ is the time, $\rho$ is the density, $\vec{v}$ is the velocity, $p$ is the thermal pressure, $\vec{B}$ is the magnetic field, $e$ is the total energy, $n$ is the number density, $\vec{F}_{c}$ is the conductive flux according to Spitzer (1962), and $\chi(T)$ is the radiative loss per unit emission measure (Colgan et al. 2008). To close the set of equations (9)-(12) we have a relation between internal, total, kinetic and magnetic energy

$$
\frac{p}{\gamma-1}=e-\frac{1}{2} \rho \vec{v}^{2}-\frac{\vec{B}^{2}}{8 \pi}
$$

where $\gamma=5 / 3$ denotes the ratio of specific heat and the expression for solar gravitational acceleration

$$
\vec{g}=-\frac{G M_{\odot}}{r^{2}} \hat{r}
$$

where $G$ is the gravitational constant, $M_{\odot}$ denotes the mass of the Sun, $r$ is the radial distance from the centre of the Sun, and $\hat{r}$ is the corresponding unit vector. We solve equations (9)-(12) with a second order TVDLF numerical scheme (Tóth \& Odstrčil 1996). In order to gain accuracy in the description of the thermal pressure, we make use of the magnetic field splitting technique (Tanaka 1994), as explained in detail in section 2.3 of Pagano et al. (2013a). The radiative losses are treated using the exact integration method (van Marle \& Keppens 2011).

The boundary conditions of the MHD simulation are treated with a system of ghost cells. Open boundary conditions are imposed at the outer boundary, reflective boundary conditions are set at the $\theta$ boundaries, and the $\phi$ boundaries are periodic. The $\theta$ boundary condition prevents any plasma or magnetic flux passing through, while the $\phi$ boundary condition allows the plasma and magnetic field to freely evolve across the boundary. These boundary conditions match those used in Mackay and van Ballegooijen (2006a) and the flux rope interacts with the $\theta$ and $\phi$ boundaries only near the end of the simulations, thus, they have no effect on the initiation and propagation of the flux rope ejection. At the lower boundary, we impose a fixed boundary condition taken from the first four $\theta-\phi$ planes of cells derived from the GNLFFF model. The computational domain is composed of $256 \times 128 \times 128$ cells distributed in a uniform grid when we use a domain extending to $4 R_{\odot}$ and $128 \times 128 \times 128$ cells when we use a domain extending to $2.5 R_{\odot}$.

\section{Simulations of an erupting flux rope}

In this section, we describe the dynamics of the flux rope when the magnetic configuration is imported from the GNLFFF model to the MPI-AMRVAC software. The aim of this section is to investigate whether the configuration is eruptive and why the flux rope ejection occurs. To this end, we adopt the simplest version of our model, where we do not include gravity and non-ideal MHD terms in the MHD equations (9)-(12), and we prescribe the plasma density distribution as in section 2.2.1 where, parameters are chosen to be $\rho_{0}=10^{-17} \mathrm{~g} / \mathrm{cm}^{3}, B_{0}=1 G$, $\rho_{\mathrm{bg}}=10^{-21} \mathrm{~g} / \mathrm{cm}^{3}$ and $p_{0}=0.006 \mathrm{dyne} / \mathrm{cm}^{2}$. 


\subsection{Initial condition}

Figure 3 illustrates the initial magnetic configuration in the MHD simulation. The magnetic field lines of the flux rope connect between the two polarities of the left-hand side (LHS) bipole (red lines, Figure 3). Overlying the flux rope, magnetic field lines create an arcade-like structure. Three additional arcade systems are also present in the initial condition: one above the right-hand side (RHS) bipole, another connecting between the two bipoles (both shown as blue lines in Figure 3 ) and finally an arcade lies high in the corona connecting between the external polarities of the bipoles (green lines, Figure 3). With the value used for the uniform pressure, and the decay of the magnetic field intensity, $\beta$ initially ranges from $10^{-3}$ near the centre of the bipoles to $10^{3}$ at the outer boundary.

To understand how the initial magnetic configuration will drive the dynamics, Figure 4 shows a map of the radial component of the Lorentz force in the $r-\phi$ plane along the centre of the bipoles. There is a strong positive (radially outward) component of the Lorentz force localized under and around the flux rope in the initial condition which pushes the flux rope outwards. We show the evolution of the system in terms of the Alfvén time, $\tau_{\text {Alf }}$, which is the travel time for an Alfvén to travel across the length of the region where the flux rope is $\tau_{\text {Alf }}=120 \mathrm{~s}$.

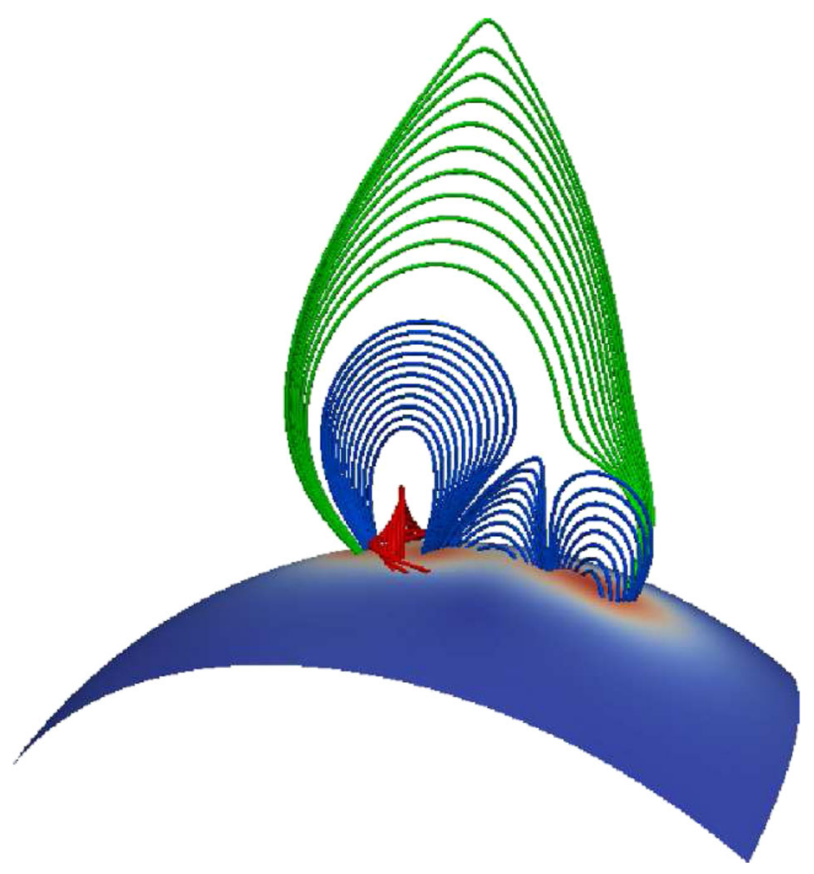

Figure 3. Magnetic field configuration used as the initial condition in the MHD simulations of the erupting flux rope. Red lines represent the flux rope, blue lines represent the arcades, and green lines represent the external magnetic field. The lower boundary is coloured according to the intensity of the magnetic field from red (strong) to blue (weak) in arbitrary units. 


\section{P. Pagano et al.}

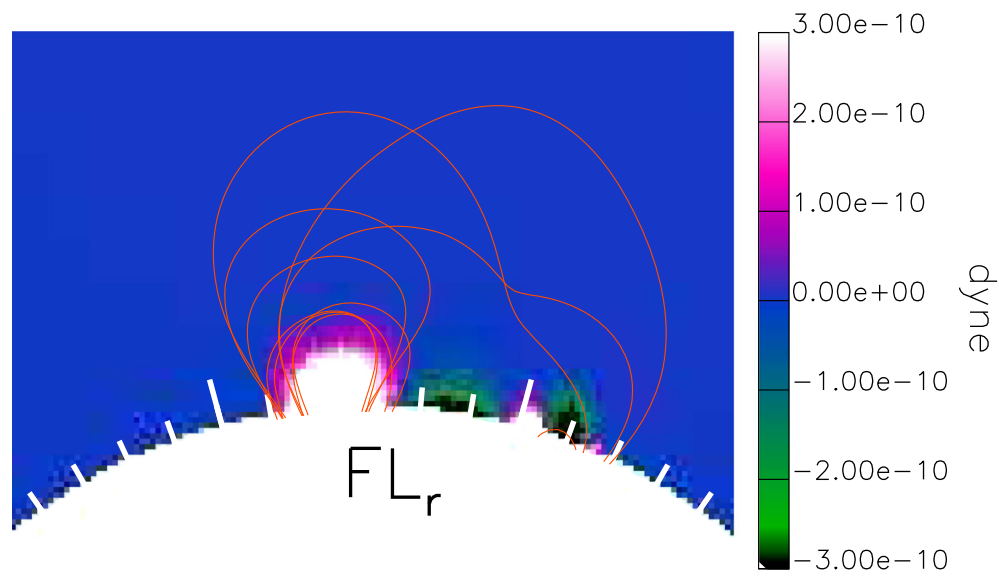

Figure 4. Radial component of the Lorentz force in an interesting portion of the $r-\phi$ plane at the centre of the bipole at $t=0 \tau_{\text {Alf }}$. Red lines represent some magnetic field lines.

\subsection{Early phase of the ejection}

At $t=0 \tau_{\mathrm{Alf}}$, the positive Lorentz force present underneath the flux rope pushes the flux rope and the plasma contained within it upwards and it immediately starts to rise. Also, the foot points of the flux rope move with a twist due to the $\theta$ and $\phi$ components of the Lorentz force. It is important to note that the upward motion is supported by a dynamic that feeds the ejection. As the flux rope moves up, a horizontal Lorentz force pushes oppositely oriented magnetic field lines on either side of the PIL towards the PIL. These magnetic field lines reconnect below the flux rope and this creates deeply dipped magnetic field lines, which increase the outward push. Figure 5 illustrates this process. In Figure 5(a) we see two individual magnetic field lines at $t=2.61 \tau_{\text {Alf }}$. These magnetic field lines are typical of the arcade magnetic field lines that connect the outer and central part of the LHS bipole.

(a)

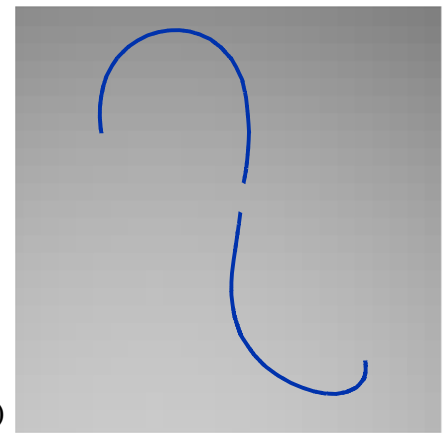

(b)

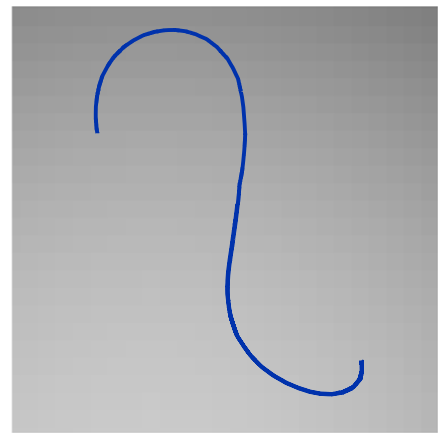

Figure 5. Magnetic field lines departing from the same points of the LHS bipole polarity regions at (a) $t=2.61 \tau_{\mathrm{Alf}}$ and (b) $t=2.67 \tau_{\mathrm{Alf}}$, viewed from the top: the flux rope develops from the upper left corner of the picture to the lower right one. 
As observed, the newly generated Lorentz force pushes these magnetic field lines towards one another, and they reconnect below the flux rope to produce a low, deeply dipped, magnetic field line underneath the flux rope (Figure $5 \mathrm{~b}, t=2.67 \tau_{\mathrm{Alf}}$ ). In this way, the radial component of the Lorentz force under the flux rope doubles in magnitude compared to its initial value.

The result is that the flux rope starts moving upwards. In Figure 6, the bulk plasma velocity at $t=11.6 \tau_{\text {Alf }}$ can be seen. Figure 6 a shows a map of the radial component of the velocity in the $r-\phi$ plane, along the centre of the bipoles, while Figure $6 \mathrm{~b}$ shows a map of the radial component of the velocity in the $\theta-\phi$ plane zoomed in at the bipoles. In addition, in Figure $6 b$, contours of the radial component of the magnetic field are shown (solid contours: positive flux, dashed: negative flux) and the green vectors denote the horizontal velocity field. In Figure $6 \mathrm{~b}$ the velocity vectors are typically of the order of $2 \times 10^{6} \mathrm{~cm} / \mathrm{s}$ while velocity vectors of magnitude

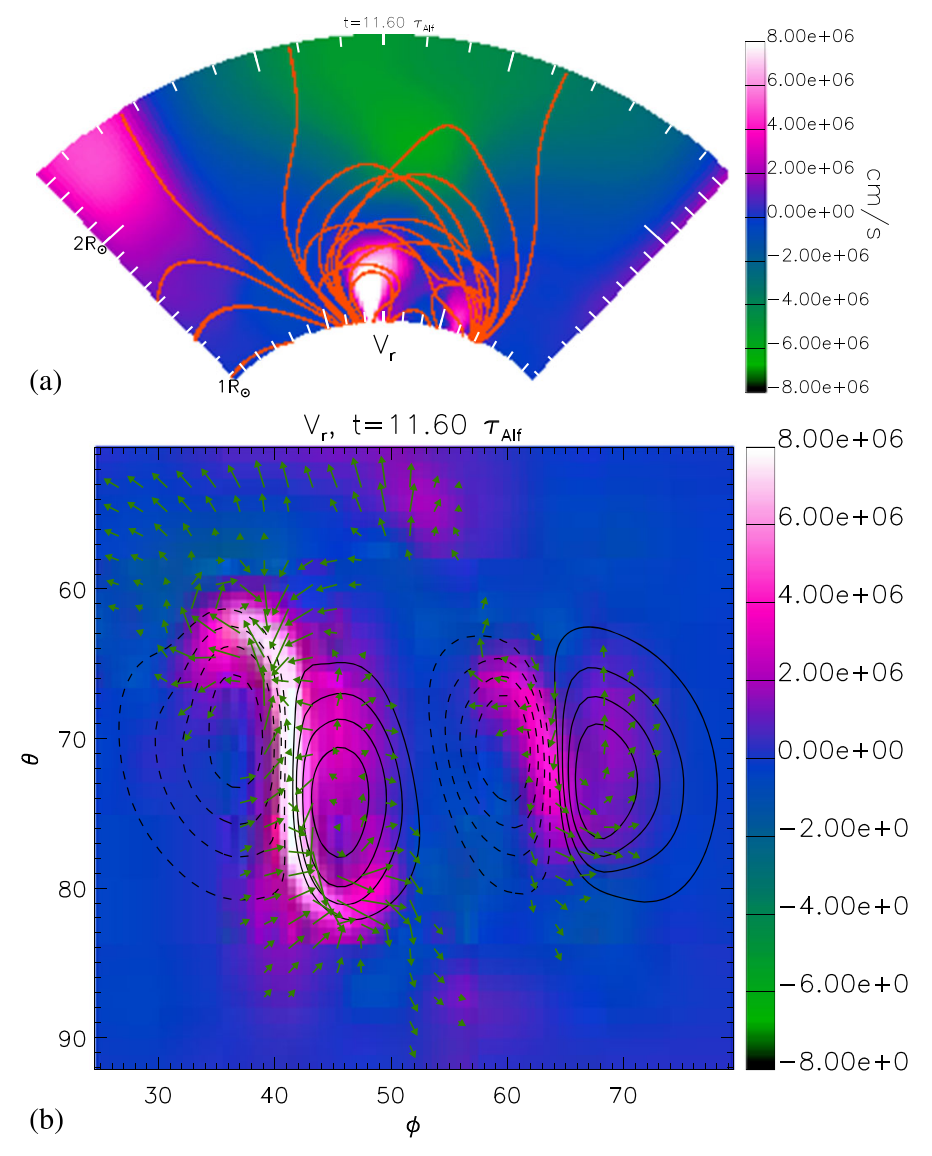

Figure 6. (a) Radial component of the velocity on the $r-\phi$ plane through the centre of the bipole at $t=11.6 \tau_{\text {Alf }}$. (b) Radial component of the velocity on the $\theta-\phi$ plane at $r=1.07 R_{\odot}$ at the same, time, with superimposed contours of the radial component of the magnetic field (black dashed lines for negative values and solid lines for positive values) and the projection of the horizontal velocity field on to the same $\theta-\phi$ plane (green arrows, typical velocity is $2 \times 10^{6}$ $\mathrm{cm} / \mathrm{s}$ and minimum velocity is $1.3 \times 10^{6} \mathrm{~cm} / \mathrm{s}$ ). 


\section{P. Pagano et al.}

lower than $1.3 \times 10^{6} \mathrm{~cm} / \mathrm{s}$ are not shown. Figure 6 a shows that the plasma above the LHS bipole is ejected outwards with a velocity higher than $8 \times 10^{6} \mathrm{~cm} / \mathrm{s}$. This motion is initially due to the positive radial Lorentz force present under the flux rope in the initial condition and, secondly, due to the reconnection of the magnetic field lines below the flux rope. Figure $6 \mathrm{~b}$ clearly shows that in the proximity of the PIL of the LHS bipole (above which the flux rope lies) the plasma moves upwards. Simultaneously, horizontal plasma motions converge towards the PIL. The convergence is most prominent in the zone around $\theta=\left[70^{\circ}, 75^{\circ}\right]$ and $\phi=\left[38^{\circ}, 42^{\circ}\right]$.

In this early phase of the dynamics, the flux rope that is ejected moves as a complete structure enclosed in the space of the arcade that lies above the LHS bipole. In the prosecution of the simulation, the flux rope keeps travelling upwards at the speed of $\sim 100 \mathrm{~km} / \mathrm{s}$, and over the simulation more complex dynamics occur, where magnetic field lines belonging to the flux rope reconnect with external field lines. However, while this simple simulation describes how the ejection occurs, it is not suitable to properly follow the propagation of the flux rope, because of the oversimplified description of the background solar corona.

\section{Parameter space investigation}

After we have described the dynamics of the early stage of the flux rope ejection and tested that the magnetic configuration under study is eruptive, we perform a parameter space investigation to identify the domain of parameters where the flux rope ejection actually turns into a CME. Specifically, we address the role of plasma $\beta$ and gravitational stratification.

In order to do so, we set-up a set of MHD simulations where we use a simplified version of the plasma distribution model in section 2.2.2, where we set $\sigma_{\text {out }}=\sigma_{\text {min }}$ to have an isothermal atmosphere, $\rho_{L B}=10^{-15} \mathrm{~g} / \mathrm{cm}^{3}$, and we solve the MHD equations (9)-(12) with solar gravity, and without non-ideal MHD terms. Moreover, we uniformly multiply the values of the component of the magnetic field of our initial configuration, so we use the maximum value of the magnetic field intensity, $B_{\max }$, as

$$
\begin{array}{c|c|c|c|}
\cline { 2 - 4 } T=3.0 \mathrm{MK} & \begin{array}{c}
1.94 \\
-1.23
\end{array} & \begin{array}{c}
0.99 \\
-2.18
\end{array} & \begin{array}{c}
0.38 \\
-2.78
\end{array} \\
\cline { 2 - 5 } \mathrm{T}=2.0 \mathrm{MK} & \begin{array}{c}
0.26 \\
-1.40
\end{array} & \begin{array}{c}
-0.70 \\
-2.36
\end{array} & \begin{array}{l}
-1.30 \\
-2.96
\end{array} \\
\cline { 2 - 5 } \mathrm{T}=1.5 \mathrm{MK} & -1.38 & -1.18 & -1.20 \\
-1.53 & -2.48 & -3.08 \\
\hline \multicolumn{2}{|c|}{\mathrm{B}_{\max }=7} & \mathrm{~B}_{\max }=21 & \mathrm{~B}_{\max }=42
\end{array}
$$

Figure 7. Table summarizing the parameter space we investigate. We ran 9 simulations with all the combinations of $T=1.5,2,3 \mathrm{MK}$ with $B_{\max }=7,21,42 \mathrm{G}$. The numbers in the cell represent the $\log _{10}(\beta)$ at the lower boundary (lower number) and the $\log _{10}(\beta)$ at the upper boundary (higher number). Red are negative values, and blue are positive ones. 


\section{Numerical Simulations of a Flux Rope Ejection}

a simulation parameter. For these simulations we extend the computational domain to $4 R_{\odot}$ using equation (2).

Thus, we run a set of nine simulations using three different temperatures $(T=1.5$, 2, $3 \mathrm{MK}$ ) of the corona and three different maximum intensity values of the magnetic field $\left(B_{\max }=7,21,42 \mathrm{G}\right)$, as illustrated in Figure 7 .

Figure 8 shows cuts of density (Figure $8 \mathrm{a}$ ) and $\beta$ (Figures $8 \mathrm{~b}$ and $8 \mathrm{c}$ ) along the radial direction through the centre of the flux rope, for some sets of simulation in order to highlight different properties of coronal atmosphere in different conditions. In the table in Figure 7, a higher corona temperature implies (i) a more uniform density and pressure gradient from the photosphere to the outer corona (Figure 8a), (ii) a higher amount of mass that constitutes the solar corona (Figure 8a), (iii) a higher $\beta$ in the outer corona (Figure $8 \mathrm{~b}, B_{\max }=7 \mathrm{G}$ case). In the lower corona $\left(r<1.2 R_{\odot}\right)$ $\beta$ is always below $\sim 10^{-1}$ regardless of the temperature, while in the outer corona switches from a low to a high $\beta$ regime when the temperature increases from 1.5 to $3 \mathrm{MK}$ (Figure $8 \mathrm{~b}$ ). Simulations with different $B_{\max }$ (Figure $8 \mathrm{c}$ ) basically differ in
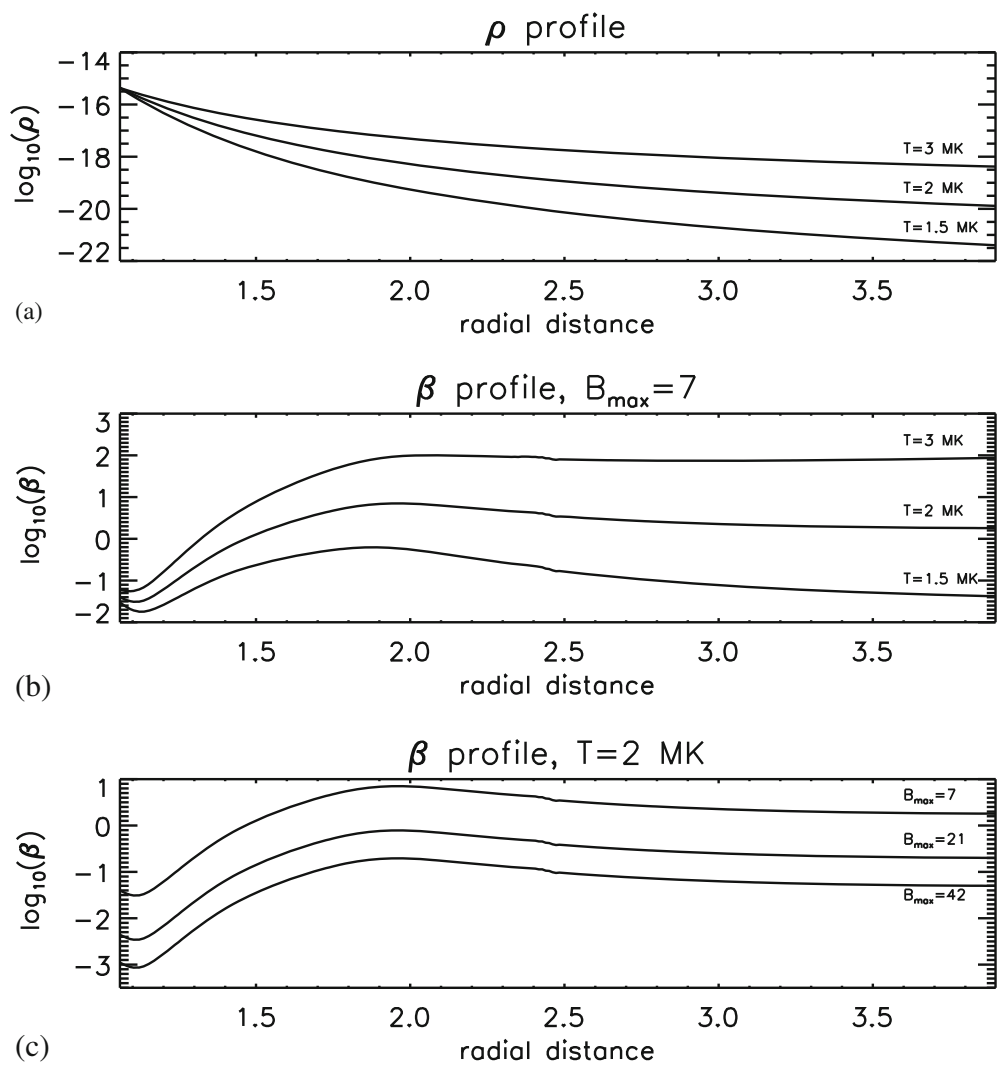

Figure 8. (a) Profiles of $\log _{10}(\rho)$ along $r$ above the centre of the LHS bipole for different temperatures of the solar corona, $T$. (b) Profiles of $\log _{10}(\beta)$ along $r$ above the centre of the LHS bipole for different temperatures of the solar corona, $T$, and with $B_{\max }=7 \mathrm{G}$. (c) Profiles of $\log _{10}(\beta)$ along $r$ above the centre of the LHS bipole with different values of the parameter $B_{\max }$ and with $T=2 \mathrm{MK}$. 


\section{P. Pagano et al.}

the plasma $\beta$, which uniformly decreases as $B_{\max }$ increases. Thus, by changing the parameter $B_{\max }$ we generally modify the dominant forces and the capacity of the solar corona plasma to react to the flux rope ejection.

In some of the simulations $\left(T=1.5 \mathrm{MK} ; B_{\max }=21\right.$ and $\left.B_{\max }=42 \mathrm{G}\right)$, we have to use a non-zero $\rho_{\text {bg }}$ (as explained in section 2.2.2) to avoid numerical problems due to extremely low $\beta$ at the external boundary. For these simulations, the background pressure and density are four orders of magnitude lower than the values near the lower boundary, where the dynamics originate and such departures from hydrostatic equilibrium only lead to appreciable effects on the magnetic configuration on time scales much longer than a flux rope ejection time scale.

In this section, we first describe the characteristics of a typical ejection in this framework, then we compare some key features between different simulations in order to highlight the role of the temperature of the stratified corona $(T)$ and the intensity of the magnetic field $\left(B_{\max }\right)$.

\subsection{Typical simulation $\left(T=2 M K, B_{\max }=21 G\right)$}

A typical simulation is one with $T=2 \mathrm{MK}$ and $B_{\max }=21 \mathrm{G}$, which is the central one in the parameter space table shown in Figure 7. In this simulation, the flux rope escapes the computational domain at $4 R_{\odot}$ and a CME occurs.

To follow the evolution of the simulation, in Figure 9 we show two quantities in the $r-\phi$ plane passing through the centre of the bipoles. Figures 9(a)-(c) show the density contrast,

$$
\rho_{c}=\frac{\rho(t)-\rho(t=0)}{\rho(t=0)},
$$

that indicates the variation in density with respect to the initial condition, which is useful for marking the ejection of plasma. Figures 9(d)-(f) show the ratio $B_{\theta} /|B|$ that illustrates the evolution of the axial magnetic field of the flux rope, which initially lies in the $\theta$-direction. As the flux rope we are investigating mostly lies on the $\mathrm{N}-\mathrm{S}$ direction, it corresponds to a region where the ratio $B_{\theta} /|B| \sim 1$ (pink and white in Figures $9(\mathrm{~d})-(\mathrm{f})$ ). As a region with opposite $B_{\theta}$ overlies the flux rope (green region in Figures 9(d)-(f)), the border of the flux rope is marked by the contour where the ratio $B_{\theta} /|B|$ changes sign.

During the simulation, the flux rope is ejected outwards, and it leads to (i) an increase in density at larger radii (Figures 9(a)-(c)), and (ii) the propagation and expansion of the region where the magnetic field is mostly axial, i.e. $B_{\theta} /|B| \sim 1$ (Figures 9(c)-(f)). It should be noted that the flux rope ejection shows the usual three component structures of the CMEs, where a higher density bow front propagates upwards, a region with lower density follows, and, finally, a dense core is located at the centre of the expanding dome. Specifically, the region where the density is higher corresponds with the region where $B_{\theta} /|B|$ remains positive too. This suggests that the magnetic flux rope is propagating upwards, perpendicular to its axial magnetic field lines, lifting coronal plasma to produce the high density region. At the same time, we can use the quantity $B_{\theta} /|B|$ to track the ejection and expansion of the CME because the region with a positive axial component of the magnetic field roughly matches the one with high-density. This is done identifying as the top of the flux rope 
the location where the quantity $B_{\theta} /|B|$ changes sign along the radial cut shown with the yellow dashed line (Figure $9(\mathrm{~d})$ ).

\subsection{Quenched ejections}

Following the location of the top of the flux rope, we track the ejection of the flux rope and, thus, to identify whether the flux rope ejection produces a CME (i.e. extends beyond $r=4 R_{\odot}$ ) or whether it is a quenched ejection. The simulations

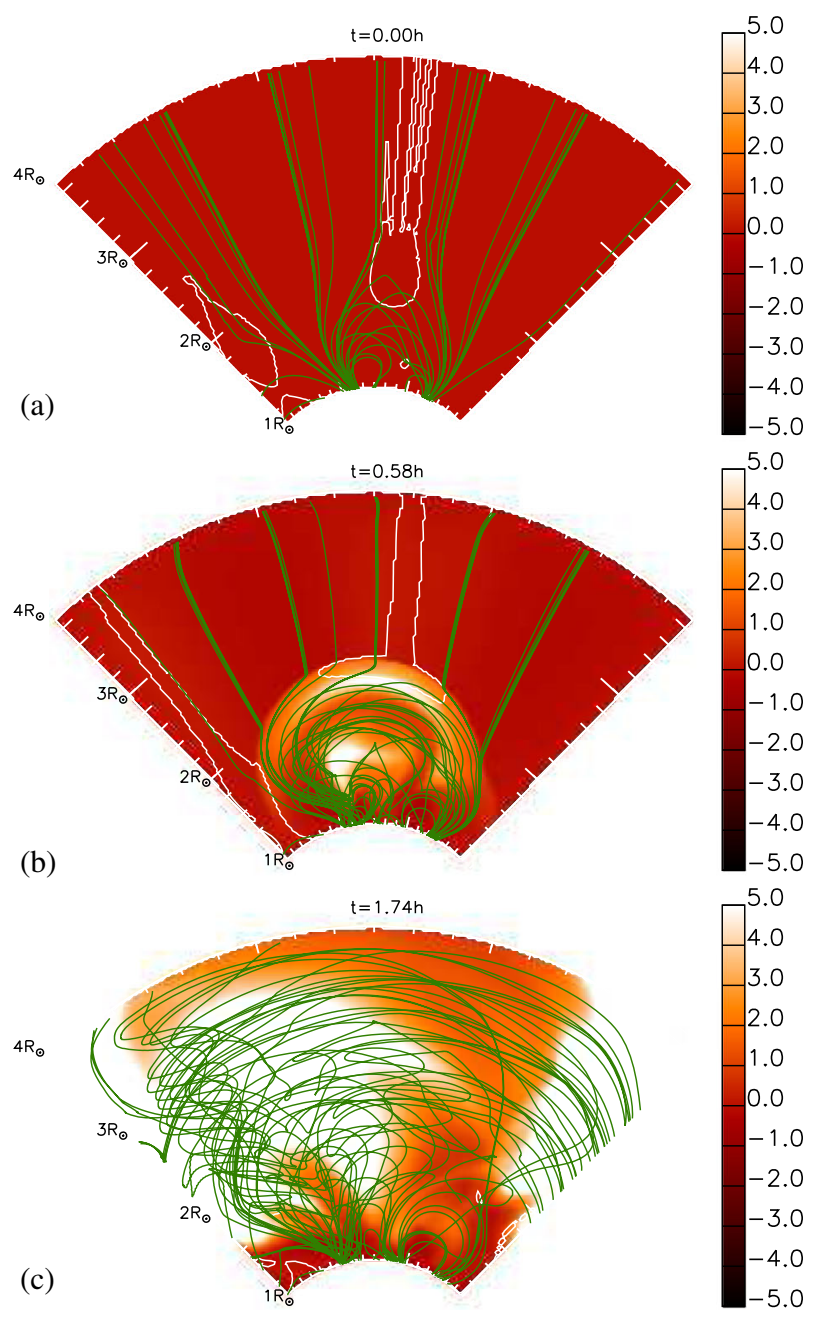

Figure 9. Simulation with $T=2 \mathrm{MK}$ and $B_{\max }=21 \mathrm{G}$. (a)-(c) Maps of density contrast (equation (15)) in the $(r-\phi)$ plane passing through the centre of the bipoles at $t=0 \mathrm{~h}, t=0.58$ $\mathrm{h}, t=1.74 \mathrm{~h}$. Superimposed are magnetic field lines plotted from the same starting points (green lines) and the contour line of $\beta=1$ (white line). (d)-(f) Maps of $B_{\theta} /|B|$ on the same plane and at the same time. Maps show the full domain of our simulations from $r=1 R_{\odot}$ to $r=4 R_{\odot}$. In panel (d), the yellow dashed line is the cut used to track the position of the top of the flux rope. 


\section{P. Pagano et al.}
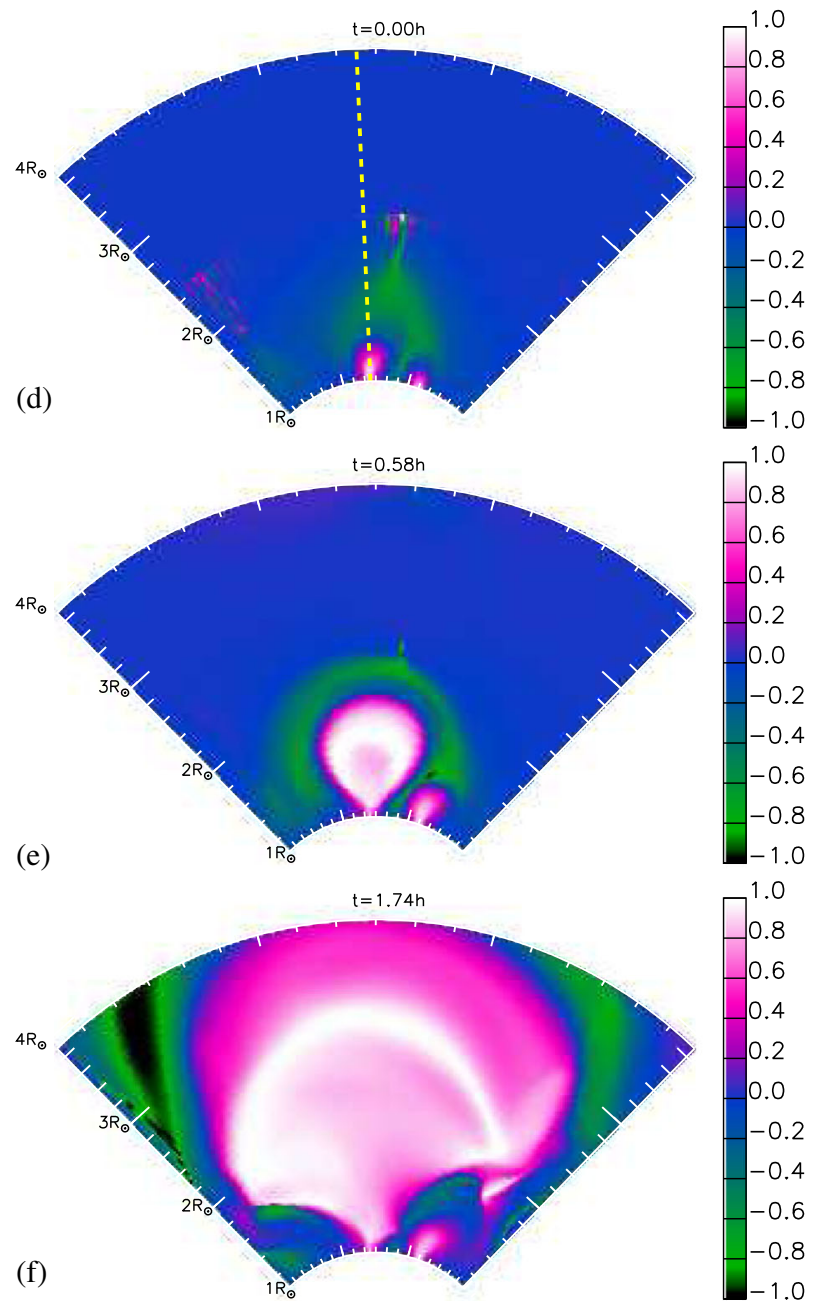

Figure 9. (continued).

with $T=2 \mathrm{MK}$ and $T=3 \mathrm{MK}$ with $B_{\max }=7 \mathrm{G}$ do not show any CME propagation, as the flux rope stops its motion shortly after the ejection. As an example, in Figure 10, we show a quenched ejection for the simulation with $T=2 \mathrm{MK}$ and $B_{\max }=7 \mathrm{G}$. In this simulation the magnetic flux rope is slowly stopped to remain positioned at $r=1.8 R_{\odot}$ after $1.74 \mathrm{~h}$. A key difference between the simulation where a CME develops (Figure 9(a)) and where the ejection is quenched (Figure 10(a)) is that a high- $\beta$ region lies above the flux rope in the simulation where the ejection is quenched. To see this, the $\beta=1$ contour (white line) overlies the flux rope in Figure 10(a), while it only surrounds the null-point region in Figure 9(a). Although this is not a necessary nor sufficient condition to determine the onset of a full or quenched ejection, whether or not a high- $\beta$ region lies over the flux rope is one of the factors that can determine the CME evolution. 

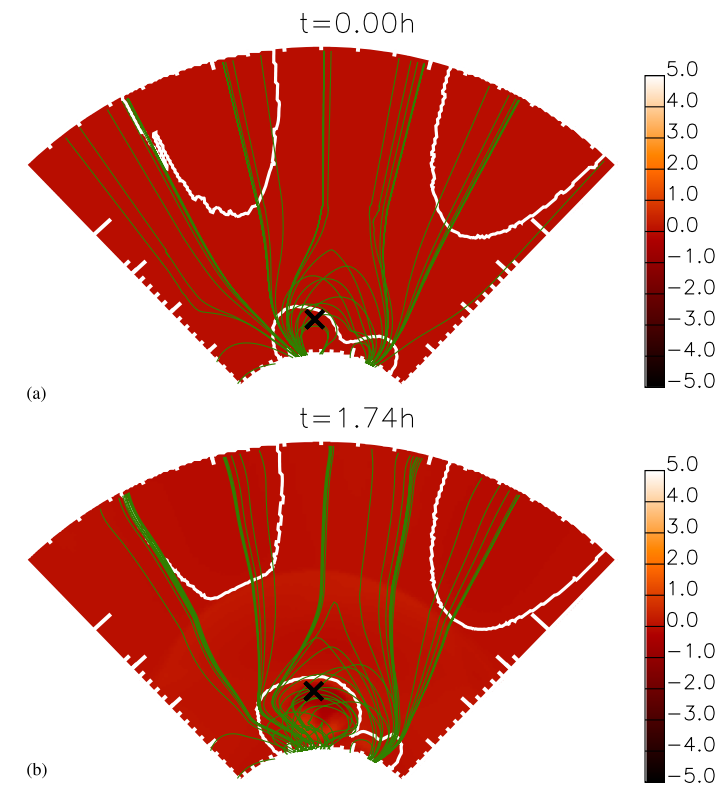

Figure 10. Simulation with $T=2 \mathrm{MK}$ and $B_{\max }=7 \mathrm{G}$. Maps of density contrast (equation (15)) in the $(r-\phi)$ plane passing through the centre of the bipoles at (a) $t=0 \mathrm{~h}$ and (b) $t=1.74 \mathrm{~h}$. Superimposed are magnetic field lines plotted from the same starting point (green lines) and the contour line of $\beta=1$ (white line). Black crosses indicate where the top of the flux rope is positioned.

\subsection{Parameter space of CMEs}

All the remaining simulations show an ejection of the flux rope in a similar manner as described in section 4.1, and here we plot the position of the top of the flux rope as a function of time. In Figure 11(a) we show the position of the top of the flux rope for the three simulations where $B_{\max }=21 \mathrm{G}$ (central column in Figure 7), until the top of the flux rope reaches the outer boundary. The speed quoted in Figure 11 is the average speed of propagation. The average speed of the CME spans a wide range from $69 \mathrm{~km} / \mathrm{s}$ to $498 \mathrm{~km} / \mathrm{s}$. The lower the temperature, the faster the CME. Note also that the CMEs propagating in a $2 \mathrm{MK}$ or $1.5 \mathrm{MK}$ corona travel at near constant speed after an impulsive acceleration phase, while the speed slightly varies in time for the slowest CME ( $T=3 \mathrm{MK}$ ). Figure 11(b) shows the height of the top of the flux rope as a function of time for the three simulations with $T=2 \mathrm{MK}$ (central row in Figure 7$)$. The higher the magnetic field $\left(B_{\max }\right)$, the larger the initial force due to the unbalanced Lorentz force under the magnetic flux rope, and the CME travels faster. With $B_{\max }=7 \mathrm{G}$, we have a quenched ejection, while we have a $718 \mathrm{~km} / \mathrm{s}$ fast CME with $B_{\max }=42 \mathrm{G}$. Note that the Alfvén speed in the system changes when the intensity of the magnetic field varies and the evolution of time scale with it. However, the result of the simulations with different $B_{\max }$ cannot be inferred solely through timescale arguments, as demonstrated by the qualitatively different evolution of the simulation with $B_{\max }=7 \mathrm{G}$ from the other simulations.

The speed at the top of the flux rope is not necessarily the speed of the CME. The top of the flux rope represents the leading edge of the CME, which is subjected to the 

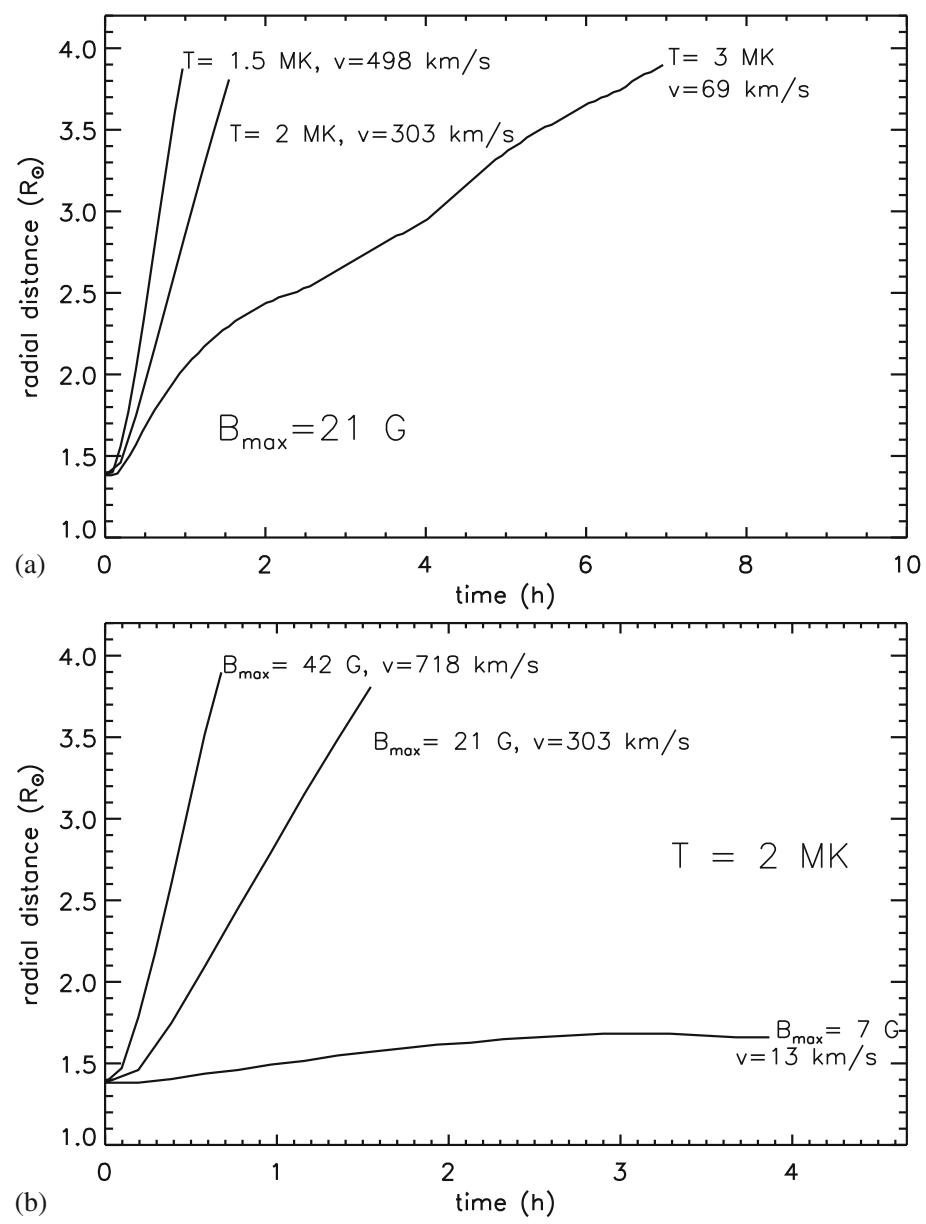

(b)

(h)

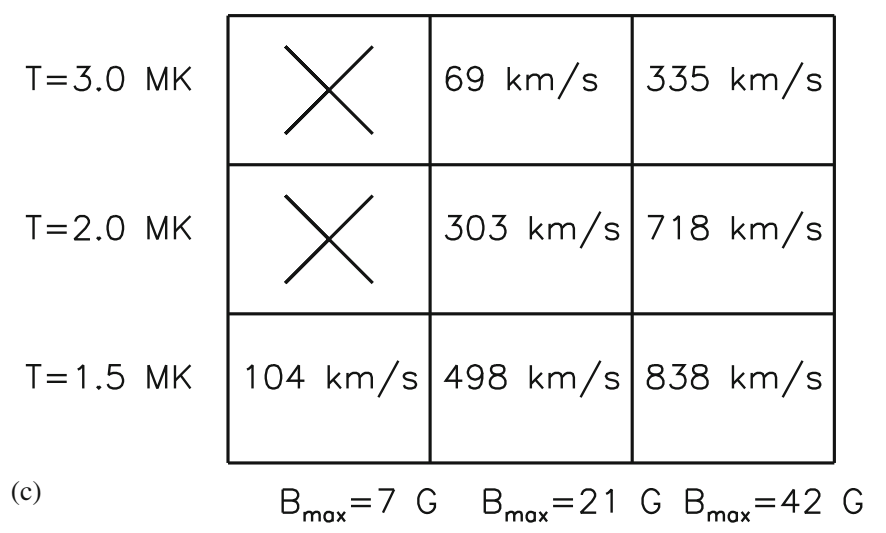

Figure 11. (a) Position of the top of the flux rope as a function of time in the three simulations with $B_{\max }=21 \mathrm{G}$. (b) Position of the top of the flux rope as function of time in the three simulations with $T=2 \mathrm{MK}$. (c) Table summarizing the average speed of the CME in the parameter space we investigate. Crosses indicate a quenched ejection and, in the other boxes, we write the value of the CME speed computed from tracking the top of the flux rope. 
combined effect of propagation and expansion. However, the results are indicative to approximately infer the speed of the CME and its dependence on the parameters $T$ and $B_{\max }$. In Figure 11(c), we summarize the speed of the CMEs for all the simulations. The fastest CME in our simulations can produce speeds of $838 \mathrm{~km} / \mathrm{s}$ and only two simulations show a quenched ejection.

\section{Synthesis of AIA observations}

As last part of our study, we develop a MHD simulation where the thermodynamics properties of the flux rope are taken into account, in order to synthesise AIA observations from our MHD simulation, and compare its main features with known observed features of flux rope ejections. We run a simulation where we solve the MHD Equations (9)-(12) with solar gravity and all the non-ideal terms listed there. We extend the computational domain to $4 R_{\odot}$ using equation (2) and we prescribe a plasma distribution based on the approach in section 2.2.2, where we choose $T_{\text {out }}=2 \mathrm{MK}, T_{\min }=10^{4} \mathrm{~K}, \rho_{L B}=3.5 \times 10^{-15} \mathrm{~g} / \mathrm{cm}^{3} B_{\max }=42 \mathrm{G}$. With these values, we obtain the atmospheric profile shown in Figure 12, where we show radial cuts of density (Figure 12(a)), thermal pressure (Figure 12(b)), and temperature (Figure 12(c)) from the lower boundary to the external boundary passing through the centre of the LHS bipole (where the flux rope lies), and a temperature profile along a cut across the center of the bipoles at the lower boundary (Figure 12(d)). In each plot, we show the corresponding hydrostatic solution at $T=2 \mathrm{MK}$ (dashed line) to highlight the properties of the atmosphere. In the density profile (Figure 12(a)), we find a decrease due to gravitational stratification, and an excess of density with respect to the hydrostatic profile below $r \sim 1.3 R_{\odot}$, where the flux rope lies above the LHS bipole. Consistently, the temperature profile (Figure 12(b)) shows a varying temperature distribution, where the flux rope is at $T \sim 10^{4} \mathrm{~K}$, cooler than the surroundings, and the external corona above the flux rope is at $T=2 \mathrm{MK}$. It should be noted that the lower boundary of the simulation is mostly at $T=2 \mathrm{MK}$ except for the locations around the PIL of the bipoles, where the temperature is lower (Figure 12(d)) and the LHS bipole shows a much lower temperature than the RHS bipole, as on the LHS bipole there is a fully formed flux rope, with more prominent axial field component, $B_{\theta}$. As explained in section 2.2.2, the thermal pressure profile (Figure 12(c)) is built to correspond to the hydrostatic profile.

\subsection{Evolution of the MHD simulation}

This simulation shows an evolution similar to what was already observed in section 3 and section 4. The evolution is illustrated in Figure 13, where maps of density and temperature are shown in the $(r-\phi)$ plane passing through the centre of the bipoles. Initially the flux rope lies near the lower boundary and, even if it is dense and heavier than the flux rope modelled in section 4 , as soon as the system is allowed to evolve the flux rope is ejected radially outwards and the high density region rises. This remains in the shape of the ejected flux rope (Figure 13(b)) initially, however, it is less identifiable as a flux rope near the end of the simulation (Figure 13(c)). After approximately 30 minutes, the front of the high density front reaches the outer boundary at $4 R_{\odot}$. 


\section{P. Pagano et al.}
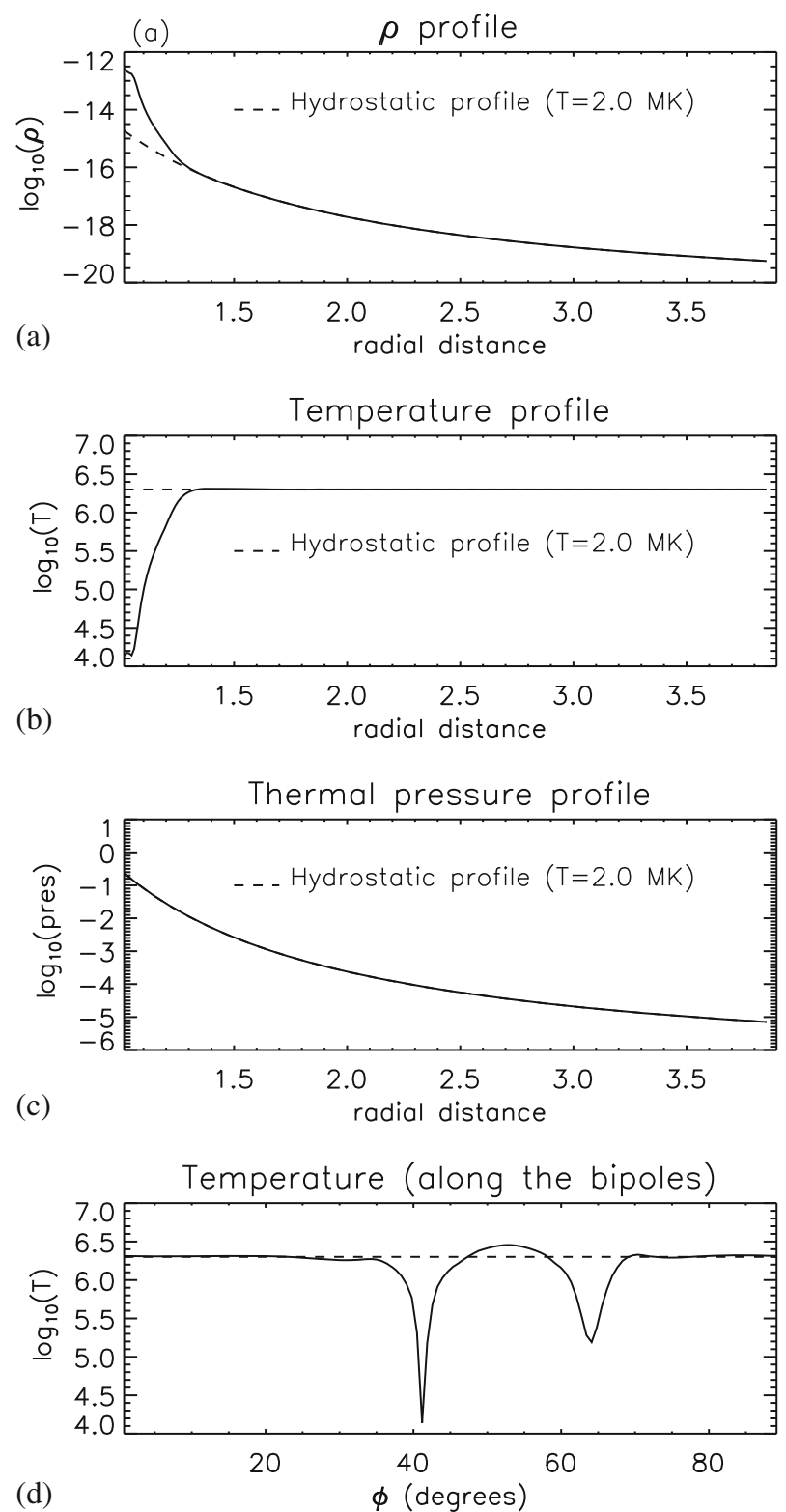

Figure 12. Profiles along the radial direction above the centre of the LHS bipole at $t=0 \mathrm{~min}$ of (a) $\log _{10}(\rho)$, (b) $\log _{10}(T),(\mathbf{c}) \log _{10}(p)$. (d) Profile along the $\phi$ direction across the center of the bipoles of $\log _{10}(T)$. The dashed lines show the equivalent profiles for a coronal atmosphere in hydrostatic equilibrium at $T=2 \mathrm{MK}$.

The flux rope is initially colder than the surrounding corona at approximately $T=10^{4} \mathrm{~K}$ (Figure 13(d), dark area), but, as soon as the ejection starts its temperature increases and a region of temperature of around $T=1 \mathrm{MK}$ is produced. This region then expands and propagates upwards and the density starts decreasing along 
with the temperature. Simultaneously, the ejection compresses the plasma ahead of it and a hot front propagates, where the temperature is about $10^{7} \mathrm{~K}$. In Figure 13(e), we find that the temperature distribution at the location of the ejection presents some variations, but a clear hot front overlies the ejection that clearly marks where the perturbation of the ejection has reached (Figure 13(f)). At the same time, the flux rope is always located at a temperature dip within the ejection front (Figures 13(e) and 13(f)). Conclusively, by comparing maps of density and temperature, we infer that in the MHD simulation the flux rope is initially denser and colder than the coronal atmosphere and preserve these properties throughout the ejection even if heating occurs due to compression and numerical resistivity.
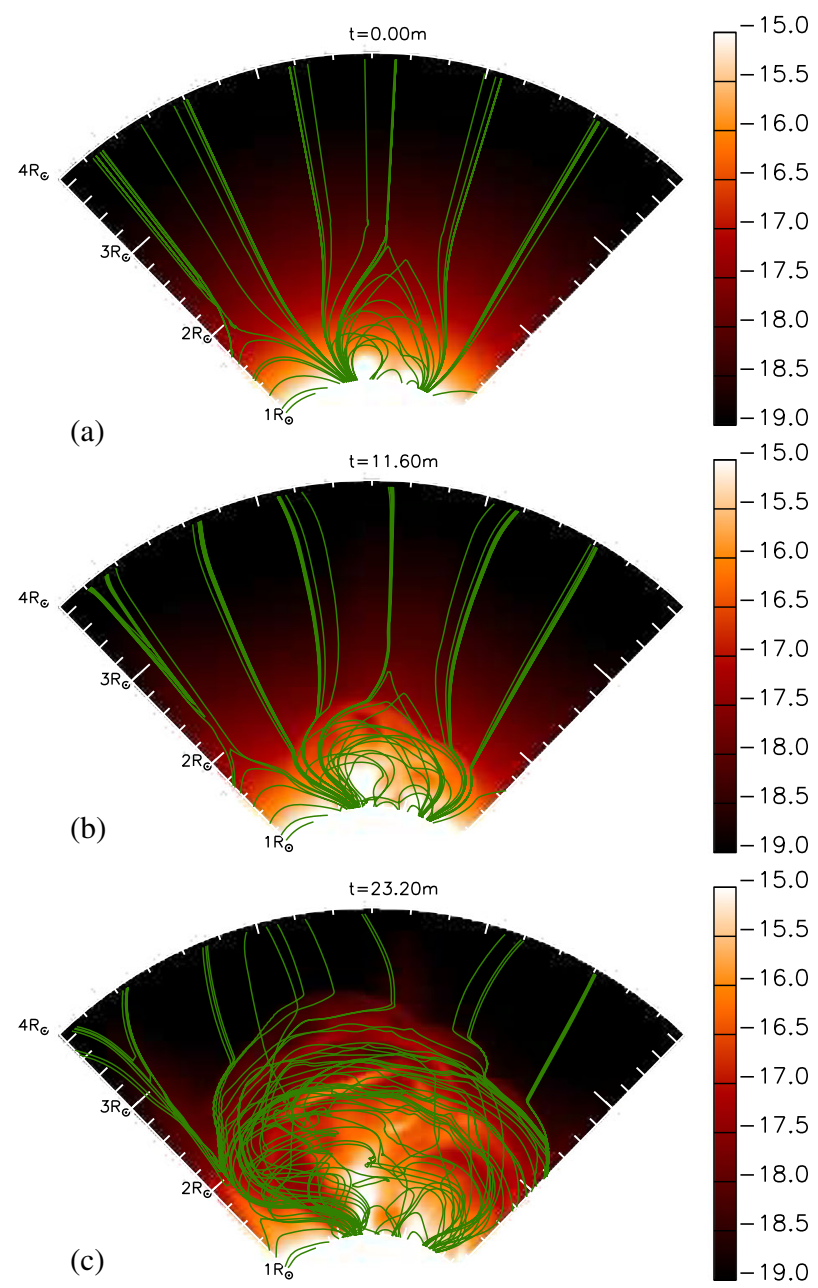

Figure 13. (a)-(c) Maps of $\log _{10}(\rho)$ in the $(r-\phi)$ plane passing through the centre of the bipoles at $t=0, t=11.60$, and $t=23.20 \mathrm{~min}$. Superimposed are magnetic field lines plotted from the same starting points (green lines). (d)-(f) $\operatorname{Maps}$ of $\log _{10}(T)$ on the same plane and at the same time. Maps show the full domain of our simulation from $r=1 R_{\odot}$ to $r=4 R_{\odot}$. 


\section{P. Pagano et al.}
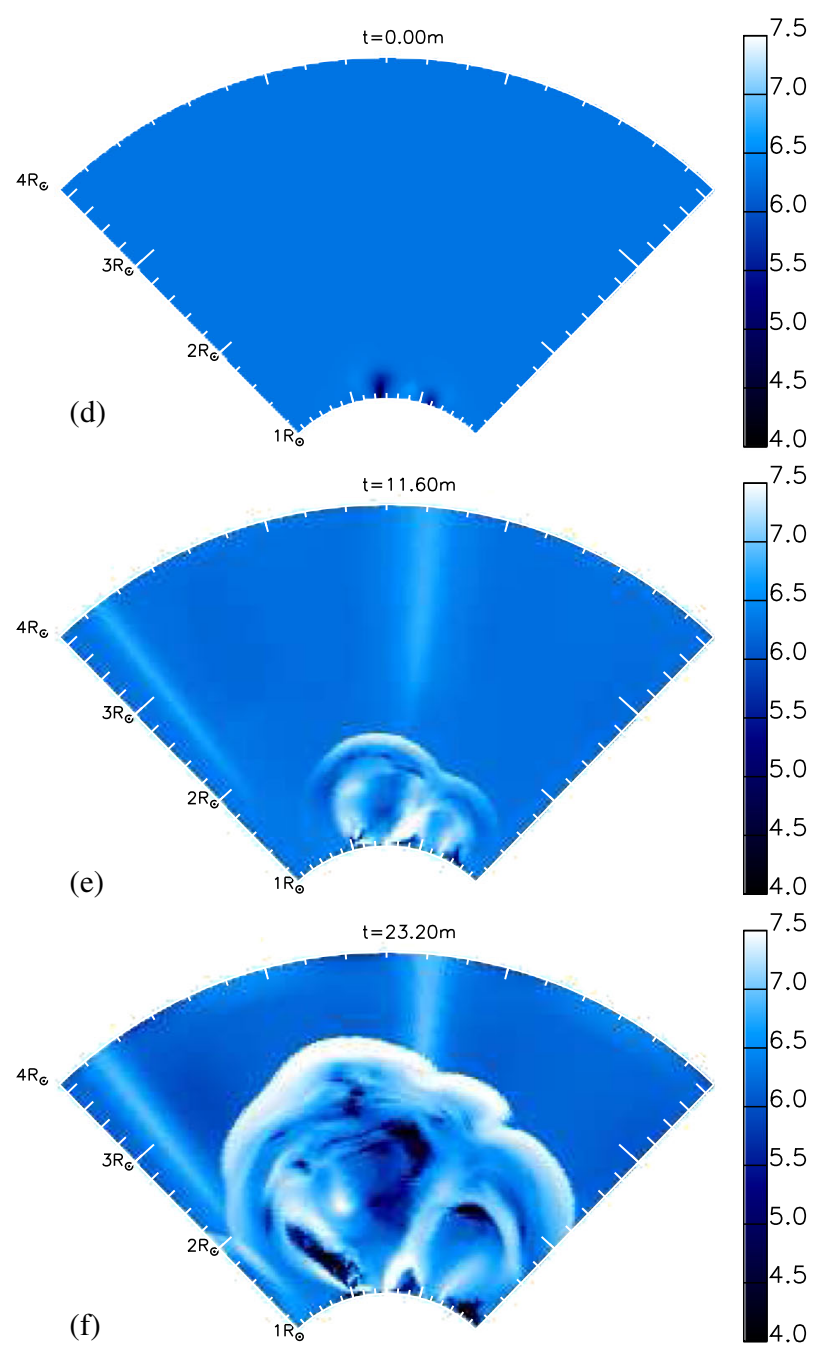

Figure 13. (continued).

\subsection{AIA emission synthesis}

We use a simple technique to synthesise the observations of AIA from our MHD simulation with the use of the AIA module in Solar Software. We follow three steps: (i) with the AIA module of the Solar Software, we create the instrumental response function for each channel as a function of temperature; (ii) we then compute the synthesized emission from each cell of the MHD simulation (interpolated in a 3D cartesian domain); (iii) finally we integrate along the line-of-sight. Therefore, we compute the emission from each element of plasma observed by each EUV channel of AIA as

$$
E M_{\text {ch. }}(n, T)=n^{2} \zeta(T)_{\text {ch. }},
$$

where $\zeta(T)_{\text {ch. }}$ is provided by the AIA module in Solar Software and in Figure 14 we show the functions $\zeta(T)_{\text {ch. }}$ for all the channels of AIA. For all channels the emission 


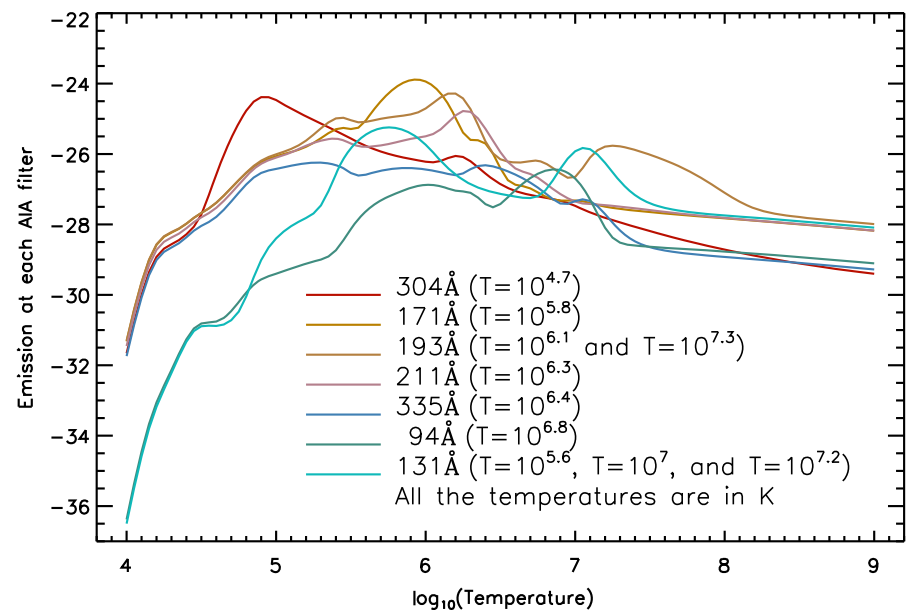

Figure 14. Values of $\log _{10}\left(\zeta(n, T)_{\text {ch. }}\right)$ for all the AIA channels as a function of $T$.

peaks far from the considered temperature extrema at $T=10^{4} \mathrm{~K}$ and $T=10^{8} \mathrm{~K}$ and our simulation produces a plasma temperature range well included within these extrema.

\subsection{Synthesized AIA observations}

We show here the synthesized observations for 2 of the AIA channels ( $171 \AA, 211 \AA$ ). These channels show the evolution of the flux rope ejection in the solar corona at commonly observed temperatures. In section 5.3.1, we describe the synthesized observations for the channels at $171 \AA$ (peak at $T \sim 10^{5.8} \mathrm{~K}$ ), observing relatively cool plasma and in section 5.3.2, we describe the synthesized observations for the channels at $211 \AA$ (peak at $T \sim 10^{6.3} \mathrm{~K}$ ) commonly used to track propagation of signals in the solar corona during flares or eruptions.

5.3.1 $171 \AA$ Channel. Figure 15 shows the synthesized observations of $171 \AA$ channel from the simulation at $t=0,11.60$ and $23.20 \mathrm{~min}$, where the flux rope initially lies on the solar surface at $30^{\circ}$ from the plane of the sky in the direction of the observer. This viewpoint has no particular symmetry and is suitable to describe a general ejection. The field-of-view used in Figure 15 is $1.5 \times 2.15 R_{\odot}$ wide and represents a portion of the disk as can be viewed by AIA. Figure 15 shows the logarithm of synthesized data numbers per second.

Initially, the flux rope is clearly visible in $171 \AA$ channel (Figure 15(a)), as this channel picks out the external shell of the flux rope being sensitive to higher temperatures than the very central axis. At $t=11.60 \mathrm{~min}$, the ejection has already occurred and the synthesized observations produce several features similar to observations. The eruption is clearly visible with an arc-shaped structure propagating outwards. The structure is already displaced from the solar surface, is slightly fragmented on its sides, and shows a small void under the arc of the ejecting flux rope, 


\section{P. Pagano et al.}

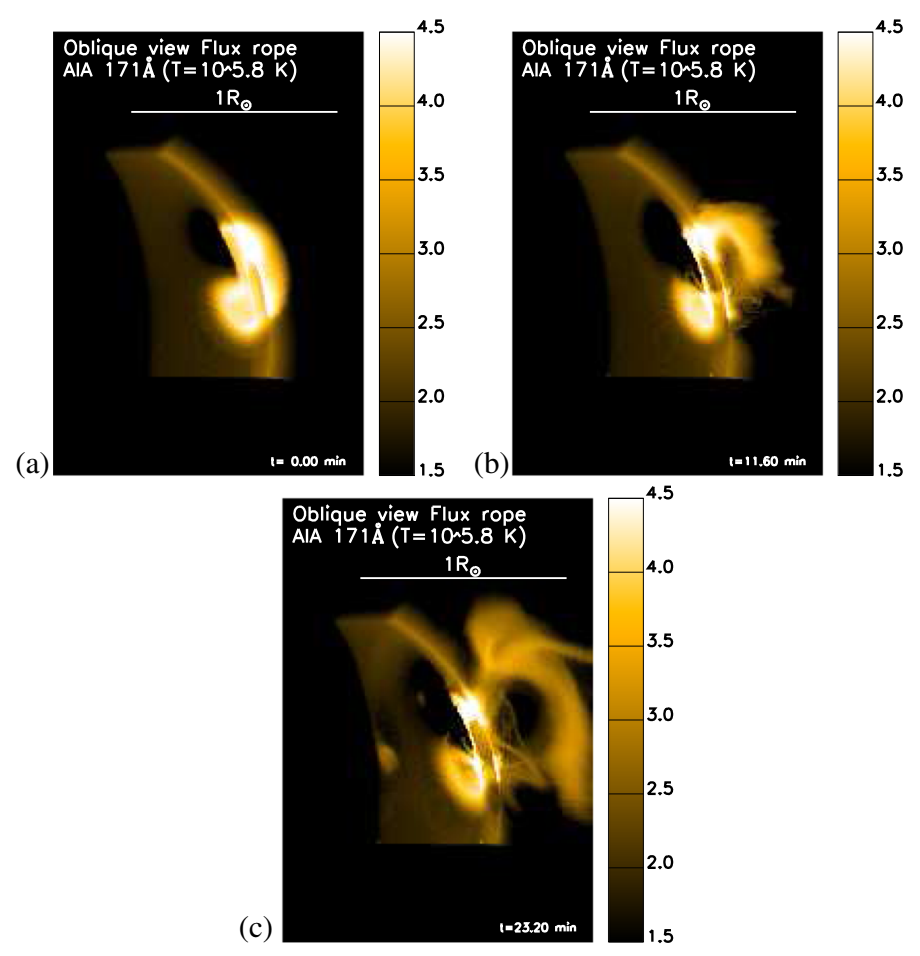

Figure 15. Synthesized observations of AIA in the $171 \AA$ channel at (a) $t=0$ min, (b) $t=11.60 \mathrm{~min}$ and (c) $t=23.20 \mathrm{~min}$. All maps show the logarithm of synthesized data numbers per second (DNS).

while its northern footpoint seems to be more evidently connected to the solar surface than the southern one. Comparing the position of the structures in Figure 15(b) and the analysis of the position of the flux rope centre, it is possible to infer that they both correspond to the ejected flux rope, as the front has already propagated off the field-of-view.

In the images at $t=23.20$ min (Fig. 15(c)), we find similar but more expanded features, as the ejected flux rope has expanded and acquired the shape of a circular ejection with a void placed at its centre. The void is quite clear and the profile near the outer edge of the ejection follows the arc shape with some features that open up because of individual bundles of magnetic field lines that reconnected with the open magnetic field. At the same time, it can be seen that the emission from the solar surface has been perturbed by the ejection. In all of the images shown in Figure 15, we show the logarithm of absolute data numbers per second (DNS) obtained in our study. These values of DNS are comparable to, within an order of magnitude, with actual off-limb observations of $171 \AA$ A channel of AIA during solar eruptions.

5.3.2 $211 \AA$. When we analyse the emission on $211 \AA$ A channel, instead, we do analyse the response of the lower corona to the flux rope ejection. To this purpose, 
we synthesize the AIA observation of a Halo CME, where the flux rope is ejected in the line-of-sight direction, as to avoid overlapping structures on the solar surface. The $211 \AA$ channel has the response peak near 2 MK, which is the temperature of

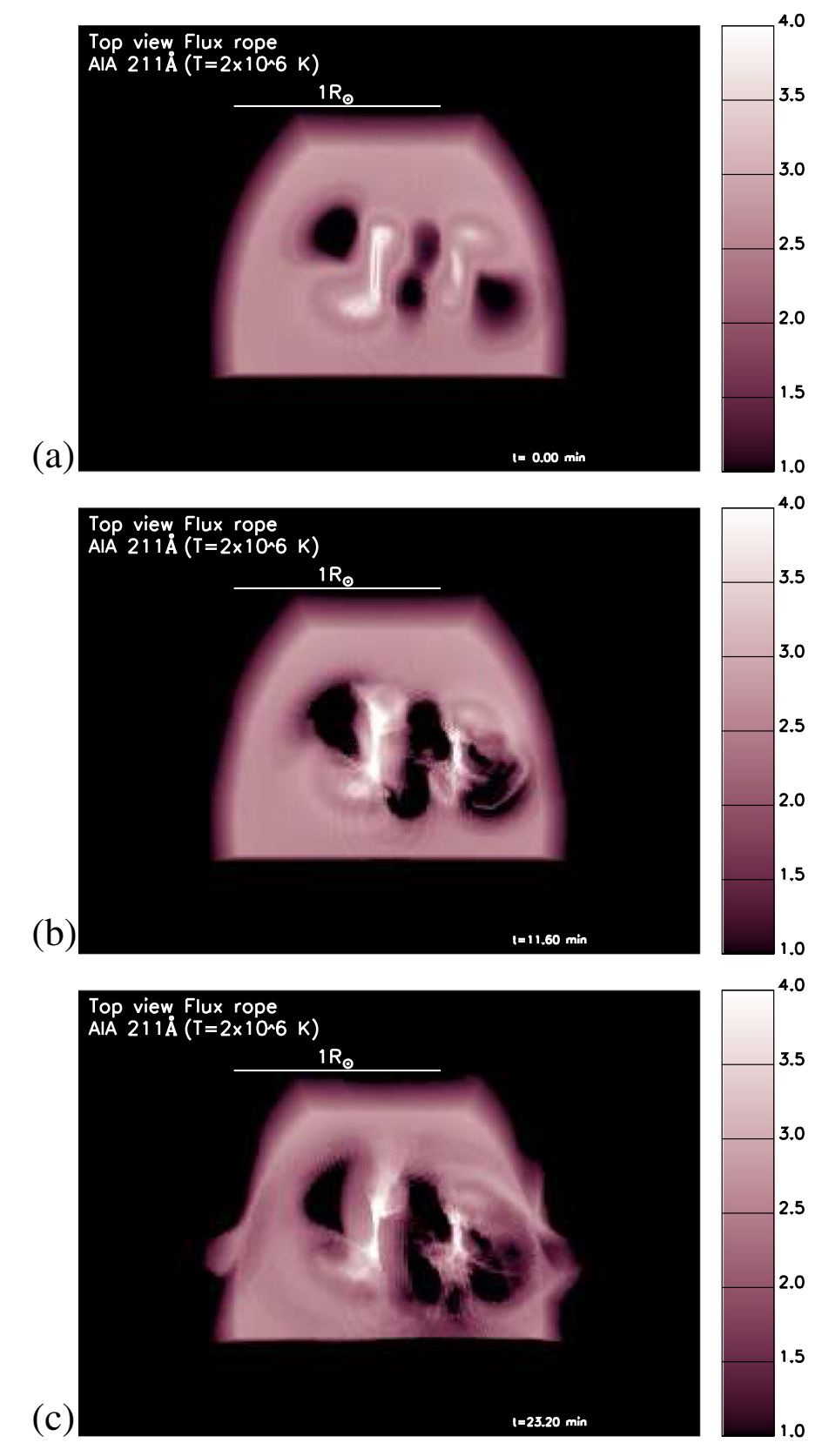

Figure 16. Synthesized observations of AIA in the $335 \AA$ and $94 \AA$ channels at (a) $t=0$ $\min ,(\mathbf{b}) t=11.60 \mathrm{~min}$ and (c) $t=23.20 \mathrm{~min}$. All maps show the logarithm of synthesized data numbers per second (DNS). The temporal evolution is available in the on-line edition. 


\section{P. Pagano et al.}

the background corona in MHD simulation and tracks well temperature and density variations of the background corona as a consequence of the flux rope ejection.

Figure 16 shows the synthesized AIA observations at $t=0 \mathrm{~min}, t=11.60 \mathrm{~min}$ and $t=23.20 \mathrm{~min}$ for the $211 \AA$ channel. At $t=0 \mathrm{~min}$, the flux rope is visible because of its high density even if the core temperature of the flux rope is far from the peak temperature of the $211 \AA$ channel (Figure 16(a)). The rest of the atmosphere is less bright than the flux rope and two dark regions are present on both sides of the flux rope because of the low density.

At $t=11.60 \mathrm{~min}$, when the ejection is already ongoing, the flux rope is still visible in the $211 \AA$ channel, although the ejection is not evident because of the projection along the line of sight effect (Figure 16(b)). The regions around the flux rope are clearly perturbed where the dark patch west from the flux rope has shrunk because of the compression of plasma, and the one east from the flux rope has slightly expanded. The borders of the bright region from where the flux rope has ejected appear modified, but not changed, in extension. Moreover, there is trace of a circular signal propagating from the south footpoint of the flux rope.

Finally, at $t=23.20 \mathrm{~min}$ (Figure 16(c)), when the flux rope has travelled far from the photosphere, the bright region has significantly shrunk and only a thin bright structure is present, corresponding to the current sheet generated by the flux rope motion. The dark regions have further shrunk and it is now possible to notice a circular front around the bipole region that corresponds to the expanding front of the ejection, which has a dome shape in 3D.

\section{Conclusions}

In this work, we are presenting a complete study on a flux rope ejection model. We start our investigation with the coupling of the GNLFFF model with a MHD numerical simulation in order to show the eruptivity of a magnetic configuration, where a flux rope has formed through the effect of differential rotation, meridional flow and surface diffusion. We then identify the parameters space of the background solar corona, where the flux rope ejection is allowed and we finally synthesise the AIA observation in $171 \AA$ and $211 \AA$ channels from a MHD simulation, where we account for the presence of the flux rope in the initial density and temperature distribution, and for non-ideal MHD terms.

To begin with, we investigate whether a flux rope formed slowly as a result of flux cancellation and photospheric shearing motions in a smoothly varying continuous magnetic field distribution can lead to a flux rope ejection that can turn into a CME. To do this, we take advantage of the coupling of two models: the first is a GNLFFF model that describes the quasi-static formation of the flux rope over time periods of days to weeks and the second is a full MHD simulation that follows the evolution of the system once the flux rope is formed. In our model, no additional shearing or stress of the magnetic field is carried out in the MHD simulation, and the resulting dynamics are solely a result of the stress built up between the two bipoles during the slow evolution in the GNLFFF model. To some extent our model, though split into two techniques, is similar to the flux cancellation model of flux rope ejection of van Ballegooijen \& Martens (1989), where the stress required for the ejection is built via photospheric motion and magnetic diffusion above the PIL. In addition to this, in our scenario, magnetic reconnection is used to produce the flux 
rope, but is not necessary in the early stages of the ejection, which is caused by a non-equilibrium.

For future studies, an important achievement of our work is the development of a technique that successfully couples two different models of the solar corona evolution. Such a technique can be applied not only to investigate the issue of flux rope ejection, but it is a useful tool that also enables us to run large scale simulation of the solar corona, needed for space weather forecast, where we can run different segments of the simulation with one or the other model, depending on the physical regime considered. Such an approach can potentially drastically reduce computational times and opens up the possibility of many future studies on the Sun's global magnetic field which would not be possible otherwise.

We also investigated the role of gravitational stratification in the propagation of CMEs, and specifically under which conditions of gravitational stratification temperature and plasma $\beta$, the flux rope ejection can turn into a CME or when it is just a quenched ejection. We do so by letting evolve an identical eruptive magnetic field configuration in a set of MHD simulations. We find that the gravitational stratification plays a role in the propagation of CMEs in the solar corona through the way it sets the plasma $\beta$. Basically, a low- $\beta$ region above the flux rope favours the ejection of the flux rope, whereas high- $\beta$ regions are more capable of reacting with the ejected flux rope and may slow down or even quench the flux rope ejection. We find that a cooler solar corona $(T \sim 1.5 \mathrm{MK})$ can help the escape of the CME and make it travel faster.

Finally, we synthesise EUV AIA observations of a flux rope ejection to compare the features present in our MHD simulations with analogous observed feateures. Our model shows a qualitative agreement between the observations of CMEs as seen by AIA and the ones synthesised from our simulations. In fact, many of the CMEs observed with AIA present a bright arcade rising in cold channels (either $171 \AA$ or $304 \AA$ ). Moreover, we also find that the south footpoint is less anchored to the photosphere than the north one, and this phenomenon can explain a diversity of shapes of flux rope ejection fronts. For instance, when one foot point is totally detached, leading to the complete reconnection of one footpoint with open magnetic flux, a different CME shape may be produced, such as a rising tail. In our setup, we have two bipoles and, asymmetries around the erupting region may explain this feature if reconnection of the magnetic field is topologically favoured at one footpoint. In addition to this, the flux rope is clearly visible from the beginning in the $171 \AA$ channel, which is common in actual AIA observations, where bright features in cold channels are usually observed where the CMEs originate. Also, the synthesis of the $211 \AA$ channel provides interesting insights which are worth investigating in future studies. The flux rope ejection is not well visible in the synthesized images because of the projection along the line-of-sight, and because this channel response function peaks at temperatures hotter than flux rope temperature. However, perturbations propagating from the flux rope ejection location towards other regions of the solar corona are clearly outlined by this channel and this is also visible in our simulations.

In future, we plan to adopt our coupling technique in a more complex study, where we start from actually observed magnetic configurations of the entire solar corona reconstructed from real magnetograms and evolved with the GNLFFF. Yeates et al. (2010) have shown that flux rope ejections can be predicted with such an approach, and we intend to couple this application of the GNLFFF model with large scale MHD 


\section{P. Pagano et al.}

simulation of the solar corona. Finally, the synthesis of AIA observations has proved to be an useful tool to bridge our modelling effort with actual observations, and we intend to replicate this approach also for other future observations, especially from solar orbiter.

\section{References}

Amari, T., Luciani, J. F., Aly, J. J., Mikic, Z., Linker, J. 2003a, ApJ, 585, 1073-1086.

Amari, T., Luciani, J. F., Aly, J. J., Mikic, Z., Linker, J. 2003b, ApJ, 595, 1231-1250.

Amari, T., Aly, J.-J., Luciani, J.-F., Mikic, Z., Linker, J. 2011, ApJl, 742, L27.

Antiochos, S. K., DeVore, C. R., Klimchuk, J. A. 1999, ApJ, 510, 485-493.

Archontis, V., Török, T. 2008, A\&A, 492, L35-L38.

Archontis, V., Hood, A. W., Tsinganos, K. 2014, ApJl, 786, L21.

Aulanier, G., Török, T., Démoulin, P., DeLuca, E. E. 2010, ApJ, 708, 314-333.

Chatterjee, P., Fan, Y. 2013, ApJl, 778, L8.

Chen, J. 1989, ApJ, 338, 453-470.

Chen, P. F. 2011, Living Reviews in Solar Physics, 8, 1.

Colgan, J., Abdallah, J. Jr., Sherrill, M. E., Foster, M., Fontes, C. J., Feldman, U. 2008, ApJ, 689, 585-592.

Fan, Y. 2005, ApJ, 630, 543-551.

Fan, Y. 2010, ApJ, 719, 728-736.

Fan, Y., Gibson, S. E. 2007, ApJ, 668, 1232-1245.

Forbes, T. G. 1991, Geophysical and Astrophysical Fluid Dynamics, 62, 15-36.

Forbes, T. G., Isenberg, P. A. 1991, ApJ, 373, 294-307.

Inoue, S., Kusano, K. 2006, ApJ, 645, 742-756.

Inoue, S., Hayashi, K., Magara, T., Choe, G. S., Park, Y. D. 2014, ApJ, 788, 182.

Jacobs, C., Poedts, S., van der Holst, B. 2006, A\&A, 450, 793-803.

Jiang, C., Feng, X., Wu, S. T., Hu, Q. 2013, ApJl, 771, L30.

Kliem, B., Török, T. 2006, Physical Review Letters, 96(25), 255002.

Kliem, B., Linton, M. G., Török, T., Karlický, M. 2010, Solar Physics, 266, 91-107.

Kliem, B., Török, T., Thompson, W. T. 2012, Solar Physics, 281, 137-166.

Kliem, B., Su, Y. N., van Ballegooijen, A. A., DeLuca, E. E. 2013, ApJ, 779, 129.

Kliem, B., Lin, J., Forbes, T. G., Priest, E. R., Török, T. 2014, ApJ, 789, 46.

Kusano, K., Maeshiro, T., Yokoyama, T., Sakurai, T. 2004, ApJ, 610, 537-549.

Kusano, K., Bamba, Y., Yamamoto, T. T., Iida, Y., Toriumi, S., Asai, A. 2012, ApJ, 760, 31.

Leake, J. E., Linton, M. G., Antiochos, S. K. 2014, ApJ, 787, 46.

Lemen, J. R., Title, A. M., Akin, D. J., Boerner, P. F., Chou, C., Drake, J. F., Duncan, D. W., Edwards, C. G., Friedlaender, F. M., Heyman, G. F., Hurlburt, N. E., Katz, N. L., Kushner, G. D., Levay, M., Lindgren, R. W., Mathur, D. P., McFeaters, E. L., Mitchell, S., Rehse, R. A., Schrijver, C. J., Springer, L. A., Stern, R. A., Tarbell, T. D., Wuelser, J.-P., Wolfson, C. J., Yanari, C., Bookbinder, J. A., Cheimets, P. N., Caldwell, D., Deluca, E. E., Gates, R., Golub, L., Park, S., Podgorski, W. A., Bush, R. I., Scherrer, P. H., Gummin, M. A., Smith, P., Auker, G., Jerram, P., Pool, P., Soufli, R., Windt, D. L., Beardsley, S., Clapp, M., Lang, J., Waltham, N. 2012, Solar Physics, 275, 17-40.

Mackay, D. H., van Ballegooijen, A. A. 2006a, ApJ, 641, 577-589.

Mackay, D. H., van Ballegooijen, A. A. 2006b, ApJ, 642, 1193-1204.

MacTaggart, D., Hood, A. W. 2009, A\&A, 508, 445-449.

Mikic, Z., Linker, J. A. 1994, ApJ, 430, 898-912.

Nishida, K., Nishizuka, N., Shibata, K. 2013, ApJl, 775, L39.

Pagano, P., Mackay, D. H., Poedts, S. 2013a, A\&A, 554, A77.

Pagano, P., Mackay, D. H., Poedts, S. 2013b, $A \& A$, 560, A38. 


\section{Numerical Simulations of a Flux Rope Ejection}

Pagano, P., Mackay, D. H., Poedts, S. 2014, A\&A, 568, A120.

Porth, O., Xia, C., Hendrix, T., Moschou, S. P., Keppens, R. 2014, ApJS, $214,4$.

Priest, E. R., Forbes, T. G. 1990, Solar Physics, 126, 319-350.

Roussev, I. I., Sokolov, I. V., Forbes, T. G., Gombosi, T. I., Lee, M. A., Sakai, J. I. 2004, ApJl, 605, L73-L76.

Roussev, I. I., Galsgaard, K., Downs, C., Lugaz, N., Sokolov, I. V., Moise, E., Lin, J. 2012, Nature Physics, 8, 845-849.

Sakurai, T. 1976, Publ. Astr. Soc. Japan, 28, 177-198.

Savcheva, A., Pariat, E., van Ballegooijen, A., Aulanier, G., DeLuca, E. 2012, ApJ, 750, 15.

Shiota, D., Kusano, K., Miyoshi, T., Shibata, K. 2010, ApJ, 718, 1305-1314.

Spitzer, L. 1962, Physics of Fully Ionized Gases. Physics of Fully Ionized Gases, Interscience (2nd edition), New York.

Tanaka, T. 1994, J. Comput. Phys., 111, 381-390.

Török, T., Kliem, B. 2005, ApJl, 630, L97-L100.

Török, T., Panasenco, O., Titov, V. S., Mikić, Z., Reeves, K. K., Velli, M., Linker, J. A., De Toma, G. 2011, ApJl, 739, L63.

Török, T., Temmer, M., Valori, G., Veronig, A. M., van Driel-Gesztelyi, L., Vršnak, B. 2013, Solar Physics, 286, 453-477.

Tóth, G., Odstrčil, D. 1996, J. Comput. Phys., 128, 82-100.

Tousey, R. 1973, The Solar Corona. In M. J. Rycroft and S. K. Runcorn, editors, Space Research, page 713.

van Ballegooijen, A. A., Martens, P. C. H. 1989, ApJ, 343, 971-984.

van der Holst, B., Keppens, R., Meliani, Z. 2008, Computer Physics Communications, 179, 617-627.

van Marle, A. J., Keppens, R. 2011, Computers \& Fluids, 42, 44-53.

Vourlidas, A., Lynch, B. J., Howard, R. A., Li, Y. 2013, Solar Physics, 284, 179-201.

Yeates, A. R., Mackay, D. H. 2009, ApJ, 699, 1024-1037.

Yeates, A. R., Attrill, G. D. R., Nandy, D., Mackay, D. H., Martens, P. C. H., van Ballegooijen, A. A. 2010, ApJ, 709, 1238-1248.

Zuccarello, F. P., Meliani, Z., Poedts, S. 2012, ApJ, 758, 117. 